Estudios Geológicos, 67(2)

julio-diciembre 2011, 333-362

ISSN: $0367-0449$

doi:10.3989/egeol.40627.192

\title{
Les Rhinocerotidae (Mammalia, Perissodactyla) miocènes et pliocènes des Tugen Hills (Kénya)
}

\author{
Miocene and Pliocene Rhinocerotidae (Mammalia, Perissodactyla) \\ of the Tugen Hills (Kenya)
}

\author{
C. Guérin ${ }^{1}$
}

RESUME

Plus de 70 restes déterminables appartenant à neuf espèces de Rhinocerotidae ont été recueillis dans 26 gisements miocènes et pliocènes des Tugen Hills, les plus riches étant Cheboit, Kapsomin, Kipsaraman et Tabarin. Ce matériel permet d'accroître sensiblement nos connaissances sur l'anatomie de ces espèces. II est désormais possible de distinguer le squelette post-crânien de Aceratherium acutirostratum de celui de Dicerorhinus leakeyi; l'anatomie crânienne et dentaire de Paradiceros mukirii est mieux connue. L'extension temporelle de Chilotheridium pattersoni et de Paradiceros mukirii sont sensiblement accrues. Ceratotherium praecox est le rhinocéros le plus fréquent à partir du Miocène terminal. Diceros bicornis est attesté depuis $6 \mathrm{Ma}$, sensiblement au même moment que les premiers $C$. praecox. Diceros cf. pachygnathus est reconnu dans les formations de Lukeino et de Mabaget, ce qui accroît considérablement l'extension géographique des espèces de ce groupe. L'association de l'Hominidé Orrorin tugenensis avec Ceratotherium praecox dans trois des quatre sites où il a été découvert (et, dans un seul site à chaque fois, avec Diceros cf. pachygnathus, D. bicornis et Brachypotherium lewisi) montre que son environnement végétal était varié mais à dominante ouverte et plutôt humide.

Mots-clés: Mammalia, Rhinocerotidae, Miocène moyen et supérieur, Pliocène, Kénya.

\begin{abstract}
More than 70 determinable and usable rhinoceros remains belonging to nine species were yielded by 26 Miocene and Pliocene sites from the Tugen Hills (Kenya), namely Cheboit, Kapsomin, Kipsaraman and Tabarin. Such a material allows a better knowledge of the anatomy of these species. It is now possible to distinguish the postcranial skeleton of Aceratherium acutirostratum from that of Dicerorhinus leakeyi. The cranial and dental anatomy of Paradiceros mukirii is better known. The temporal extension of Chilotheridium pattersoni and Paradiceros mukirii are increased. Ceratotherium praecox is the most frequent rhino from the Uppermost Miocene onwards. Diceros bicornis is known since $6 \mathrm{My}$, about at the same time the first $C$. praecox. Diceros cf. pachygnathus is identified in the Lukeino and Mabaget Formations, and the geographical extension of this group of species is much increased. The Hominid Orrorin tugenensis is associated with rhinoceroses in four sites of the Lukeino Formation; Ceratotherium praecox is present in three of them, alone in Kapcheberek, associated one time (Tabarin) with D. bicornis and in another one (Cheboit) with Brachypotherium lewisi; at Aragai the sole rhino is Diceros cf. pachygnathus. All these rhinos contribute to assign to Orrorin a variable but mostly open and rather wet palaeoenvironment.
\end{abstract}

Keywords: Mammalia, Rhinocerotidae, Middle and Upper Miocene, Pliocene, Kenya.

\section{Introduction}

Parmi les nombreux gisements fossilifères découverts et fouillés par la Kenya Palaeontology Expedi- tion, dirigée par B. Senut et M. Pickford, dans le Miocène et le Pliocène ancien (entre 15,5 et 4,5 Ma) de la région des Tugen Hills, 26 ont livré des Rhinocerotidae appartenant à 9 espèces. Leur répar-

\footnotetext{
1 UMR CNRS 5276 UCB Lyon 1-ENS et Département des Sciences de la Terre, Université Claude Bernard-Lyon I, 27-43 Boulevard
} du 11 novembre 1918, F 69622 Villeurbanne Cédex France. Email: Claude.Guerin @univ-lyon1.fr 
Tableau 1.-Répartition spatio-temporelle des Rhinocerotidae néogènes des Tugen Hills

\begin{tabular}{|c|c|c|c|c|c|c|c|c|c|c|c|c|}
\hline Age & Site & Formation & $\begin{array}{l}\text { Acerath. } \\
\text { acutiros. }\end{array}$ & $\begin{array}{l}\text { Brachyp. } \\
\text { heinzelini }\end{array}$ & $\begin{array}{c}\text { Brachyp. } \\
\text { lewisi }\end{array}$ & $\begin{array}{l}\text { Chiloth. } \\
\text { pattersoni }\end{array}$ & $\begin{array}{c}\text { Dicerorh. } \\
\text { leakeyi }\end{array}$ & $\begin{array}{l}\text { Paradic. } \\
\text { mukirii }\end{array}$ & $\begin{array}{l}\text { Ceratot. } \\
\text { praecox }\end{array}$ & $\begin{array}{l}\text { Diceros } \\
\text { cf. pachyg. }\end{array}$ & $\begin{array}{l}\text { Diceros } \\
\text { bicornis }\end{array}$ & $\begin{array}{l}\text { Rhino. } \\
\text { indet. }\end{array}$ \\
\hline 4,5 & Kibingor & Mabaget & & & & & & & $\mathrm{X}$ & & & \\
\hline $5-4,5$ & Mabaget & Mabaget & & & & & & & & & & $\mathrm{X}$ \\
\hline $5-4,5$ & Kapchebrit & Mabaget & & & & & & & $\mathrm{X}$ & & $\mathrm{X}$ & \\
\hline $5-4,5$ & Kinego & Mabaget & & & & & & & $\mathrm{X}$ & & & \\
\hline $5-4,5$ & Mosionin & Mabaget & & & & & & & $?$ & & $\mathrm{X}$ & \\
\hline $5-4,5$ & Ngetabkwony & Mabaget & & & & & & & $\mathrm{X}$ & $\mathrm{X}$ & & \\
\hline $5-4,5$ & Sagatia & Mabaget & & & $\mathrm{X}$ & & & & $\mathrm{X}$ & & $\mathrm{X}$ & \\
\hline 5 & Tabarin & Mabaget & & & & & & & $\mathrm{X}$ & & $\mathrm{X}$ & \\
\hline 5,2 & Kapcharar & Kapairaina & & & & & & & $\mathrm{X}$ & & & \\
\hline 5,7 & Kabongongoi & Lukeino & & & & & & & & & & $\mathrm{X}$ \\
\hline 5,7 & Koimogul & Lukeino & & & & & & & $\mathrm{X}$ & & & \\
\hline $5,8-5,7$ & Kapsomin & Lukeino & & & $\mathrm{X}$ & & & & $\mathrm{X}$ & & & \\
\hline 5,8 & Kapcheberek & Lukeino & & & & & & & $\mathrm{X}$ & & & $\mathrm{X}$ \\
\hline 6 & Kapchepkweren & Lukeino & & & & & & & $\mathrm{X}$ & & & \\
\hline 6 & Cheboit & Lukeino & & & $\mathrm{X}$ & & & & $\mathrm{X}$ & & & \\
\hline 6 & Aragai & Lukeino & & & & & & & & $\mathrm{X}$ & & \\
\hline 6 & Mutuko & Lukeino & & & & & & & & & $\mathrm{X}$ & \\
\hline 9,5 & Ainopchorit & Ngerngerwa & & & & $\mathrm{X}$ & & & & & & \\
\hline 12,5 & Merepmaina & Ngorora & & & & & $\mathrm{X}$ & & & & & \\
\hline 12,5 & Kabarsero & Ngorora & $\mathrm{X}$ & & & & & & & & & \\
\hline 13 & Bartule & Ngorora & & & & & & & & & & $\mathrm{X}$ \\
\hline 13 & Grildain & Ngorora & $\mathrm{X}$ & & & & & & & & & \\
\hline 13 & Tibenonin & Ngorora & & & & & & & & & & $\mathrm{X}$ \\
\hline $15,5-15$ & Kipsaraman & Muruyur & $\mathrm{X}$ & & & & $\mathrm{X}$ & & & & & \\
\hline 15,5 & Kaperon & Muruyur & & & & & $X$ & & & & & \\
\hline 15,5 & Cheparawa & Muruyur & $\mathrm{X}$ & $\mathrm{X}$ & & & & $\mathrm{X}$ & & & & \\
\hline
\end{tabular}

tition spatio-temporelle est précisée tabl. 1, les âges absolus m'ont été aimablement communiqués par M. Pickford.

Les restes recueillis sont abondants mais un bon nombre (phalanges, petits fragments d'os des pattes, fragments de dents) n'est pas déterminable au niveau spécifique ou même générique. Plus de 70 fossiles ont toutefois pu être déterminés et étudiés.

La richesse en rhinocéros des différents sites est très variable; les plus remarquables de ce point de vue sont Kipsaraman, Cheboit, Kapsomin et Tabarin.

Quatre gisements appartenant tous à la Formation de Lukeino, Aragai, Cheboit, Kapcheberek et Kapsomin, ont livré des restes de l'Hominidé Orrorin tugenensis (Senut et al., 2001; Pickford \& Senut, 2001). Les Rhinocerotidae qu'ils contiennent contribuent à la connaissance du paléoenvironnement de ce dernier.

Les 9 espèces de Rhinocerotidae présentes dans le Miocène et le Pliocène des Tugen Hills appartiennent à 5 des 6 lignées de Rhinocérotidés reconnues jusqu'ici en Afrique (Guérin, 2000, 2003); ces lignées qui correspondent à autant de sous-familles sont assimilées à des tribus par d'autres auteurs. Il s'agit des Dicerorhininae, des Brachypotheriinae, des Chilotheriinae, des Aceratheriinae et des Dicerotinae, cette dernière incluant les deux espèces africaines actuelles. Les Iranotheriinae, attestés dans le Miocène inférieur d'Ouganda et dans le Miocène moyen récent du Kénya (Guérin \& Pickford, 2003), ne sont connus dans la région qu'avec Kenyatherium bishopi Aguirre \& Guérin, 1974 défini dans le Miocène supérieur de Nakali au Kénya (9,5 Ma) et attesté dans les Samburu Hills, de même âge (Nakaya et al., 1999).

\section{Etude systématique}

\section{FAMILLE RHINOCEROTIDAE GRAY, 1821 \\ Genre Dicerorhinus Gloger, 1841 \\ Dicerorhinus leakeyi Hooijer, 1966}

D. leakeyi appartient à la sous-famille des Dicerorhininae, qui regroupe des rhinocéros bicornes tridactyles à face longue, de taille petite à grande, largement répandus dans le Néogène et le Quaternaire d'Eurasie, et qui survit actuellement avec $D$. sumatrensis. D. leakeyi est son principal représentant en Afrique, continent où le genre n'a fait que de brèves incursions: on connaît ainsi une forme particulière du D. hemitoechus (Fal- 

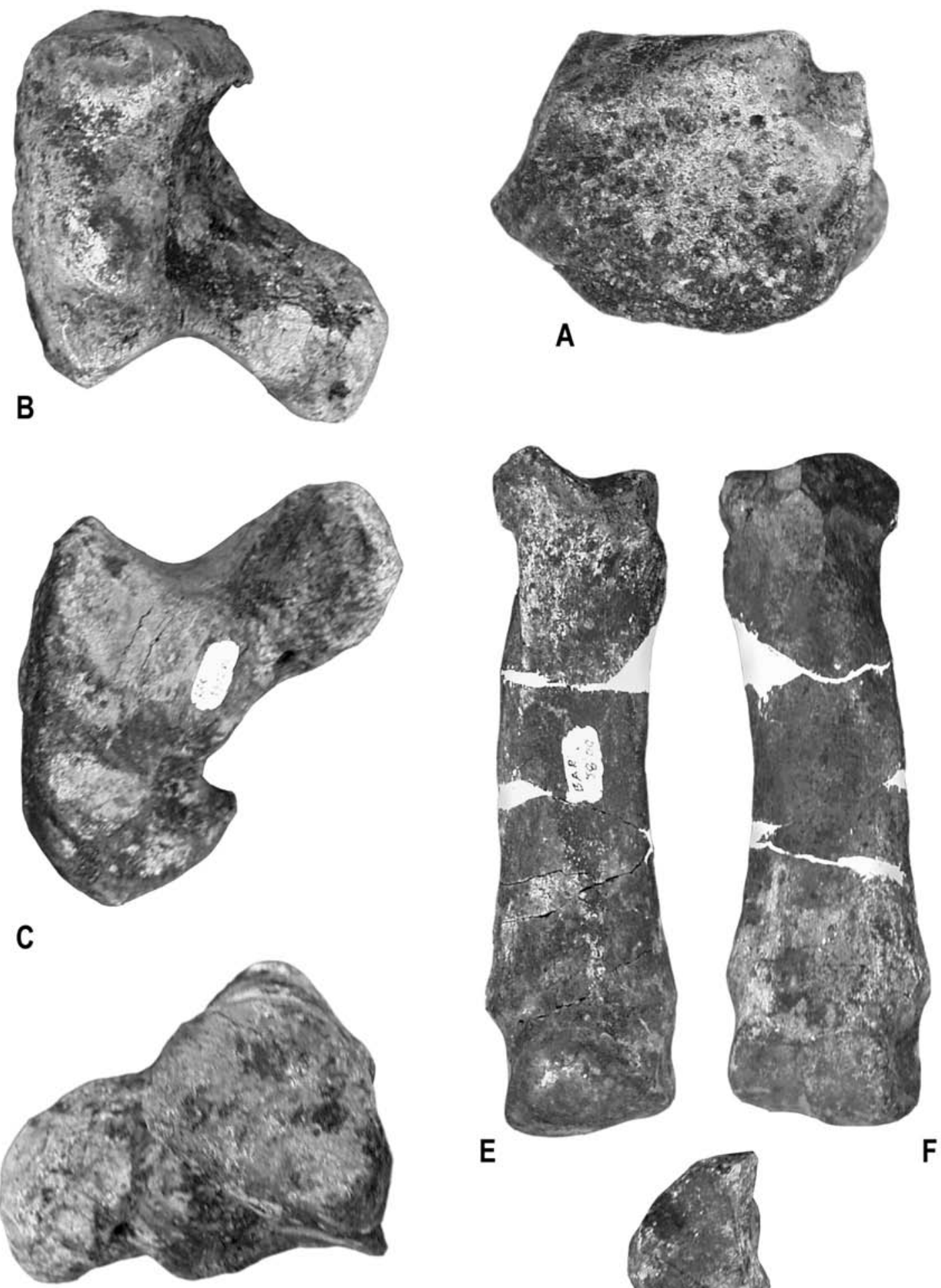

E

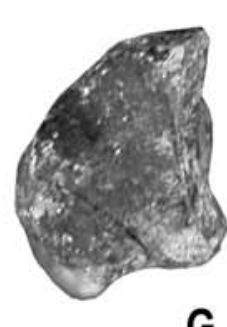

$\mathbf{F}$

D

$5 \mathrm{~cm}$

G

Fig. 1.-Brachypotherium lewisi et Dicerorhinus leakeyi. A-D: B. lewisi de Kapsomin, onciforme gauche ${ }^{\circ}$ BAR 1902'01; A: vue de face; B: vue distale; C: vue proximale; D: vue médiale. E-G: D. leakeyi de Kipsaraman, métacarpien II droit $n^{\circ}$ BAR 98'00; E: vue de face; F: vue postérieure; G: vue proximale. 
Tableau 2.-Dimensions comparées des crânes des Rhinocerotidae néogènes des Tugen Hills

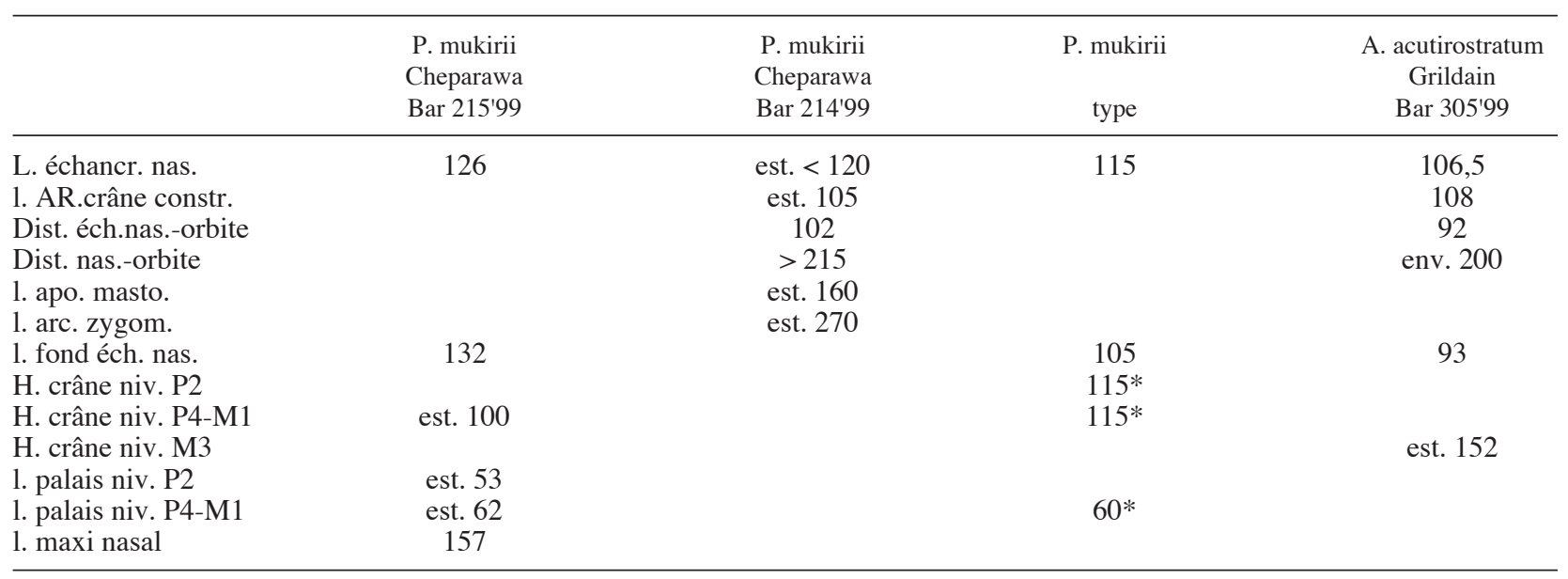

coner) européen dans le Pléistocène du Maghreb, une espèce nouvelle encore non décrite dans le Pliocène du Tchad (Koro Toro, 3,5 à 3 Ma: Likius, 2002), D. africanus Arambourg, 1970, du Villafranchien du Lac Ichkeul en Tunisie et peut-être Dicerorhinus primaevus Arambourg dans le Miocène de l'Oued el Hamman en Algérie, mais l'attribution générique de ce dernier est contestée (Geraads, 1986). Les I/2 de D. leakeyi sont développées en puissantes défenses, alors qu'elle tendront à disparaître dans une sous-lignée du Pléistocène européen, qui acquerra en même temps une ossification de la cloison nasale et l'hypsodontie des dents jugales; pendant le Néogène les jugales restent brachyodontes. Dans de nombreuses espèces les jugales supérieures, particulièrement les deux dernières prémolaires, présentent un ectolophe doté de deux puissants plis verticaux correspondant au paracône et au métacône.

Ginsburg (1974) a proposé de placer une des plus anciennes espèces du genre Dicerorhinus, D. sansaniensis (Lartet, 1851) du Miocène moyen et supérieur d'Europe, dans un genre particulier, Lartetotherium, et Groves (1983) y range aussi D. leakeyi. Les similitudes crâniales, dentaires et post-crâniennes de ces deux espèces avec l'actuel $D$. sumatrensis, espèce-type de Dicerorhinus, l'emportent beaucoup trop sur les différences pour que je suive cette opinion, et je considère Lartetotherium comme un synonyme récent de Dicerorhinus.

Diagnose spécifique originelle (traduite, légèrement simplifiée): Dicerorhinus avec cornes nasale et frontale. Incisives supérieures réduites; petites incisives entre les canines inférieures (sic! il s'agit bien évidemment des I/2). Processus inférieurs du squamosal fusionnés en dessous du pseudo-méat auditif. Occiput aussi élevé que chez D. sansaniensis. Bord inférieur de la mandibule à peu près droit comme chez $D$. sansaniensis, et non recourbé vers le haut dans sa partie symphysaire comme pour $D$. schleiermacheri, $D$. orientalis ou $D$. ringstroe$m i$. La taille du crâne est semblable à celle de D. schleiermacheri et $D$. orientalis, supérieure à celle de $D$. sansaniensis et inférieure à celle de $D$. ringstroemi. La taille des dents est intermédiaire entre $D$. sansaniensis et $D$. schleiermacheri. Prémolaires supérieures à protolophe et métalophe unis côté interne jusqu'à $15 \mathrm{~mm}$ au dessus du collet, cingulum faible, protocône sans véritable constriction. Molaires supérieures à vallée interne large et basse, cingulum interne très faible ou absent, constriction du protocône très faible ou nulle, anticrochet non proéminent, ectolophe déprimé entre les racines, crochet et crista faibles ou absents.

Holotype: un crâne avec sa mandibule.

Autre matériel originellement rapporté: un autre crâne avec sa mandibule; diverses dents isolées de Rusinga et de Napak; un squelette en connexion, endommagé et incomplet, de Rusinga.

\section{Gisement-type: Rusinga, Kénya.}

Autres gisements: outre Rusinga (18 Ma), D. leakeyi est attesté dans 5 autres gisements du Kénya: Alengerr Beds (14 à $12 \mathrm{Ma}$ ), Chemeron Formation (Miocène terminal ou Pliocène basal), Karungu (18 Ma), Maboko (15,5 Ma), Ombo (18 Ma), ainsi qu'à Napak V et VI (19,5 Ma) en Ouganda.

Remarque: en 1966 D.A. Hooijer avait estimé que le squelette post-crânien de $D$. leakeyi ne pouvait être distingué de celui de son contemporain Aceratherium acutirostratum. De ce fait le matériel post-crânien n'est jamais déterminé en collection, mais il me paraissait évident que la discrimination des deux rhinocéros ne poserait plus de problème le jour où l'on disposerait de suffisamment de matériel et d'un squelette en connexion (Guérin, 2000, 2003). En 2002 j'ai pu étudier au National Museums of Kenya à Nairobi les pattes postérieures du squelette partiel en connexion de $D$. leakeyi trouvé à Rusinga et étudié par Hooijer en 1966 (la technique de mesure de ce dernier est trop différente de la mienne pour que je puisse exploiter les dimensions qu'il a publiées). Comme on pouvait s'y attendre, les dimensions et proportions de leurs os sont suffisamment caractéristiques pour attribuer facilement à $D$. leakeyi l'un des deux lots reconnaissables parmi les restes jusqu'ici indéterminés; l'autre lot correspond alors à A. acutirostratum, ce qui est vérifié par les similitudes de proportions avec les acérathères du Miocène d'Europe. De plus, connaissant les rapports de dimensions entre certains os de la patte arrière et leurs homologues de la patte avant (les métapodes par exemple) pour une série de 15 à 20 squelettes adultes en connexion de Dicerorhinus sumatrensis, on peut à partir des métatarsiens de D. leakeyi prédire une fourchette de dimensions pour chaque métacarpien. Et un des lots jusqu'ici indéterminés correspond parfaitement à ces valeurs prédictibles. 
Tableau 3.-Dimensions comparées des jugales supérieures des Rhinocerotidae néogènes des Tugen Hills

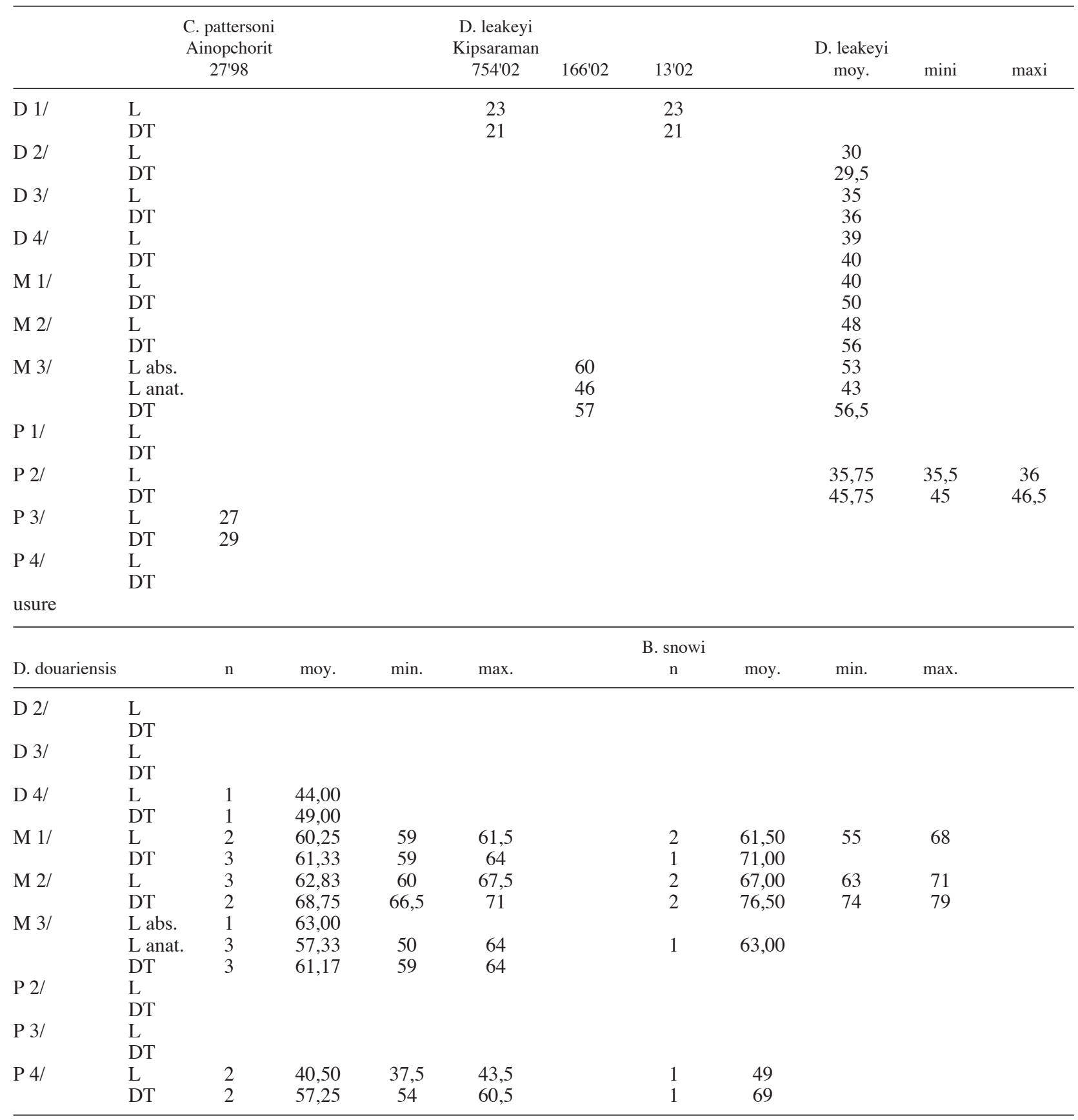

Gisements des Tugen Hills: Kaperon (formation de Muruyur, 15,5 Ma). Kipsaraman (formation de Muruyur, 15,5 - 15 Ma). Merepmaina (formation de Ngorora, 12,5 Ma).

Matériel: un fragment de mandibule portant $\mathrm{P} / 3, \mathrm{n}^{\circ} \mathrm{BAR}$ 71'00 (Merepmaina); trois jugales supérieures de Kipsaraman, $\mathrm{n}^{\circ} \mathrm{BAR} 754^{\prime} 02$ (D1/g), 13'02 (D1/ d), 166'02 (M3/g); deux I/1 vestigiales $n^{\circ}$ BAR 1076 et 1077'02 (Kipsaraman); 11 jugales inférieures de Kipsaraman, n BAR 1079’02 (D/1 g),
6’02 (D/1 g), 815’01 (D/2 g), 8'01 (D/3 g), 2099'01 (D/3), $1073^{\prime} 02$ (M/2 g), 1034'99 (P/2 d), 744'01 (P/2 d), 755'02 (P/4 g), 1058'99 (P/4 g), 1034'01 (P/4 g); épiphyse distale de radius $\mathrm{n}^{\circ}$ BAR 853'99 (Kaperon); Mc II droit complet (Fig. 1 E-G) n ${ }^{\circ}$ BAR 98'00 (Kipsaraman).

Description: Le fragment de branche horizontale de mandibule est, au niveau de l'intervalle $\mathrm{P} / 4-\mathrm{M} / 1$, haut de $63 \mathrm{~mm}$ pour un diamètre transversal de $47 \mathrm{~mm}$. Si cette dernière dimension 
Tableau 4.-Dimensions comparées des rangées dentaires supérieures des Rhinocerotidae néogènes des Tugen Hills

\begin{tabular}{|c|c|c|c|c|c|c|c|c|c|c|c|c|c|}
\hline & & $\begin{array}{c}\text { Par. mukirii } \\
\text { Cheparawa } \\
215^{\prime} 99\end{array}$ & $214^{\prime} 99$ & $214^{\prime} 99$ & & $\begin{array}{l}\text { A. acutir. } \\
\text { Chepa. } \\
665^{\prime} 99\end{array}$ & $\begin{array}{l}\text { Kaba. } \\
933^{\prime} 02\end{array}$ & $\begin{array}{c}\text { D. cf. pachyg. } \\
\text { Aragai } \\
1857^{\prime} 00\end{array}$ & $\begin{array}{l}\text { C. praecox } \\
\text { Tabarin } \\
313^{\prime} 99\end{array}$ & $\begin{array}{c}1904 \text { à } \\
1908^{\prime} 01\end{array}$ & Kapsomim & terrain & \\
\hline & & & $\mathrm{D}$ & G & & & & & & & & & \\
\hline \multirow[t]{2}{*}{ D $1 /$} & $\mathrm{L}$ & & & & & 31,5 & & & & & & & \\
\hline & DT & & & & & 24,5 & & & & & & & \\
\hline \multirow[t]{2}{*}{ D 2/ } & $\mathrm{L}$ & & & & & 42,5 & & & & & & & \\
\hline & DT & & & & & 41,5 & & & & & & & \\
\hline \multirow[t]{2}{*}{ D 3/ } & $\mathrm{L}$ & & & & & 49 & & & & & & & \\
\hline & DT & & & & & 44,5 & & & & & & & \\
\hline \multirow[t]{2}{*}{ D 4/ } & $\mathrm{L}$ & & & & & 60,5 & & & & & & & \\
\hline & DT & & & & & 54 & & & & & & & \\
\hline \multirow[t]{2}{*}{ M 1/ } & $\mathrm{L}$ & & 39 & 39 & & & 59,5 & & & & & & \\
\hline & DT & & 44,7 & 44 & & & 64 & & & & & & \\
\hline \multirow[t]{2}{*}{ M 2/ } & $\mathrm{L}$ & 39 & 43 & 43 & & & & & & 70,5 & & 59 & \\
\hline & DT & 46 & 44 & 45,5 & & & & & & 77,5 & & 79 & \\
\hline \multirow[t]{3}{*}{ M 3/ } & L abs. & 47,5 & 46,5 & 44,3 & & & & 70 & & & & & \\
\hline & $\mathrm{L}$ anat. & 38 & 34 & $33^{*}$ & & & & 58 & & & & & \\
\hline & DT & 44,5 & 42,5 & 43 & & & & 70,5 & & & & & \\
\hline \multirow[t]{2}{*}{ P 1/ } & $\mathrm{L}$ & & & & & & & & & & 32,5 & & \\
\hline & DT & & & & & & & & & & 28 & & \\
\hline \multirow[t]{2}{*}{ P 2/ } & $\mathrm{L}$ & & & 25,5 & & & & & 34 & & & & \\
\hline & DT & & & 30 & & & & & 43,5 & & & & \\
\hline \multirow[t]{2}{*}{ P 3/ } & $\mathrm{L}$ & & 32,3 & 31 & & & & & & 50 & 47 & & \\
\hline & DT & & 39,7 & 39 & & & & & & 60 & 58 & & \\
\hline \multirow[t]{2}{*}{ P 4/ } & $\mathrm{L}$ & 31 & 35 & 35 & & & & & & 50 & & & \\
\hline & DT & 45 & 41 & 42,5 & & & & & & 70 & & & \\
\hline L. RD & & 166 & est. 178 & & & & & & & & & & \\
\hline $\mathrm{L}$ des $\mathrm{P}$ & & & & 87 & & & & & & & & & \\
\hline L P3/-4/ & & 51 & 63 & 63 & & & & & & & & & \\
\hline $\mathrm{L}$ des $\mathrm{M}$ & & 104 & 113 & 114 & & & & & & & & & \\
\hline $\begin{array}{l}\text { Ceratot } \\
\text { praecox }\end{array}$ & & $\mathrm{n}$ & moy. & $\min$. & $\max$ & e-type & c. var. & $\begin{array}{c}\text { A. } \\
\text { campbelli }\end{array}$ & & & $\begin{array}{l}\text { Par. mukirii } \\
\text { Fort Ternan }\end{array}$ & & \\
\hline \multirow[t]{2}{*}{ D $1 /$} & $\mathrm{L}$ & 2 & 25,00 & 23,5 & 26,5 & & & & & & & & \\
\hline & DT & 2 & 23,25 & 21,5 & 25 & & & & & & & & \\
\hline \multirow[t]{2}{*}{ D 2/ } & $\mathrm{L}$ & 3 & 38,67 & 38 & 39,5 & & & & & & 20 & 17 & \\
\hline & DT & 3 & 37,83 & 36,5 & 39 & & & & & & & 15,5 & 16 \\
\hline \multirow[t]{2}{*}{ D 3/ } & $\mathrm{L}$ & 1 & 51,00 & & & & & & & & & 26,5 & \\
\hline & DT & 1 & 45,00 & & & & & & & & & 28,5 & \\
\hline D 4/ & $\mathrm{L}$ & 1 & 56,50 & & & & & & & & & 34,5 & \\
\hline & DT & 1 & 52,00 & & & & & & & & & 32 & \\
\hline M 1/ & $\mathrm{L}$ & 8 & 57,50 & 48 & 66 & 6,19 & 10,77 & 58 & & & & 36 & \\
\hline & DT & 13 & 68,46 & 64 & 73 & 2,43 & 3,55 & 68,5 & & & & 36,5 & \\
\hline M 2/ & $\mathrm{L}$ & 8 & 67,94 & 60 & 72 & 4,27 & 6,29 & & & & & & \\
\hline & DT & 11 & 71,05 & 65 & 77,5 & 4,38 & 6,16 & & & & & & \\
\hline M 3/ & L abs. & 10 & 76,25 & 63 & 82 & 5,46 & 7,16 & 54 & & & & & \\
\hline & $\mathrm{L}$ anat. & 9 & 65,22 & 58 & 69,5 & 4,21 & 6,46 & 47,5 & & & & & \\
\hline & DT & 11 & 63,82 & 58 & 70 & 3,63 & 5,69 & 49,5 & & & & & \\
\hline P 1/ & $\mathrm{L}$ & 2 & 27,00 & 24 & 30 & & & & & & & & \\
\hline & DT & 2 & 30,00 & 25,5 & 34,5 & & & & & & & & \\
\hline P 2/ & $\mathrm{L}$ & 8 & 39,88 & 35 & 43,5 & 3,66 & 9,18 & & & & & & 28 \\
\hline & DT & 7 & 38,86 & 36,5 & 42,5 & 1,99 & 5,13 & & & & & & 32,5 \\
\hline P 3/ & $\mathrm{L}$ & 10 & 46,80 & 40 & 50,5 & 3,65 & 7,79 & 48 & & & & 33,5 & \\
\hline & DT & 10 & 56,80 & 42,5 & 64 & 6,26 & 11,03 & 63 & & & & 41,5 & \\
\hline P 4/ & $\mathrm{L}$ & 11 & 51,59 & 45 & 55 & 3,02 & 5,84 & & & & & 31,5 & \\
\hline & DT & 10 & 64,05 & 58 & 68,5 & 3,61 & 5,63 & & & & & 45 & \\
\hline L. RD & & 3 & 311,00 & 298 & 327 & & & & & & & & \\
\hline $\mathrm{L}$ des $\mathrm{P}$ & & 5 & 134,60 & 123 & 142 & 7,54 & 5,60 & & & & & & \\
\hline L P3/-4/ & & 13 & 92,19 & 81,5 & 100,5 & 6,98 & 7,58 & & & & & 59,5 & \\
\hline $\mathrm{L}$ des $\mathrm{M}$ & & 7 & 185,21 & 176,5 & 202 & 8,31 & 4,49 & & & & & & \\
\hline
\end{tabular}




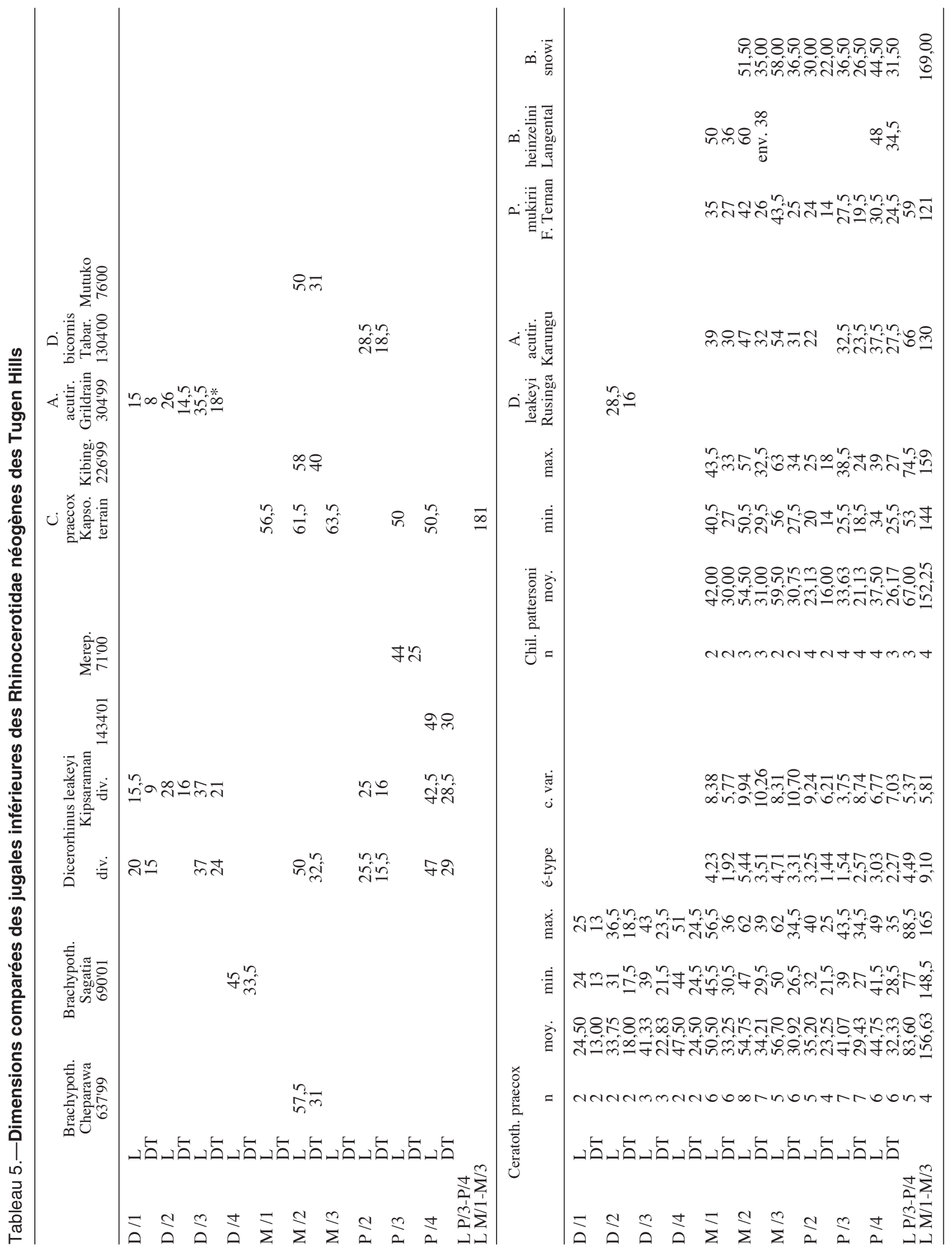


Tableau 6.-Dimensions comparées du radius des Rhinocerotidae néogènes des Tugen Hills

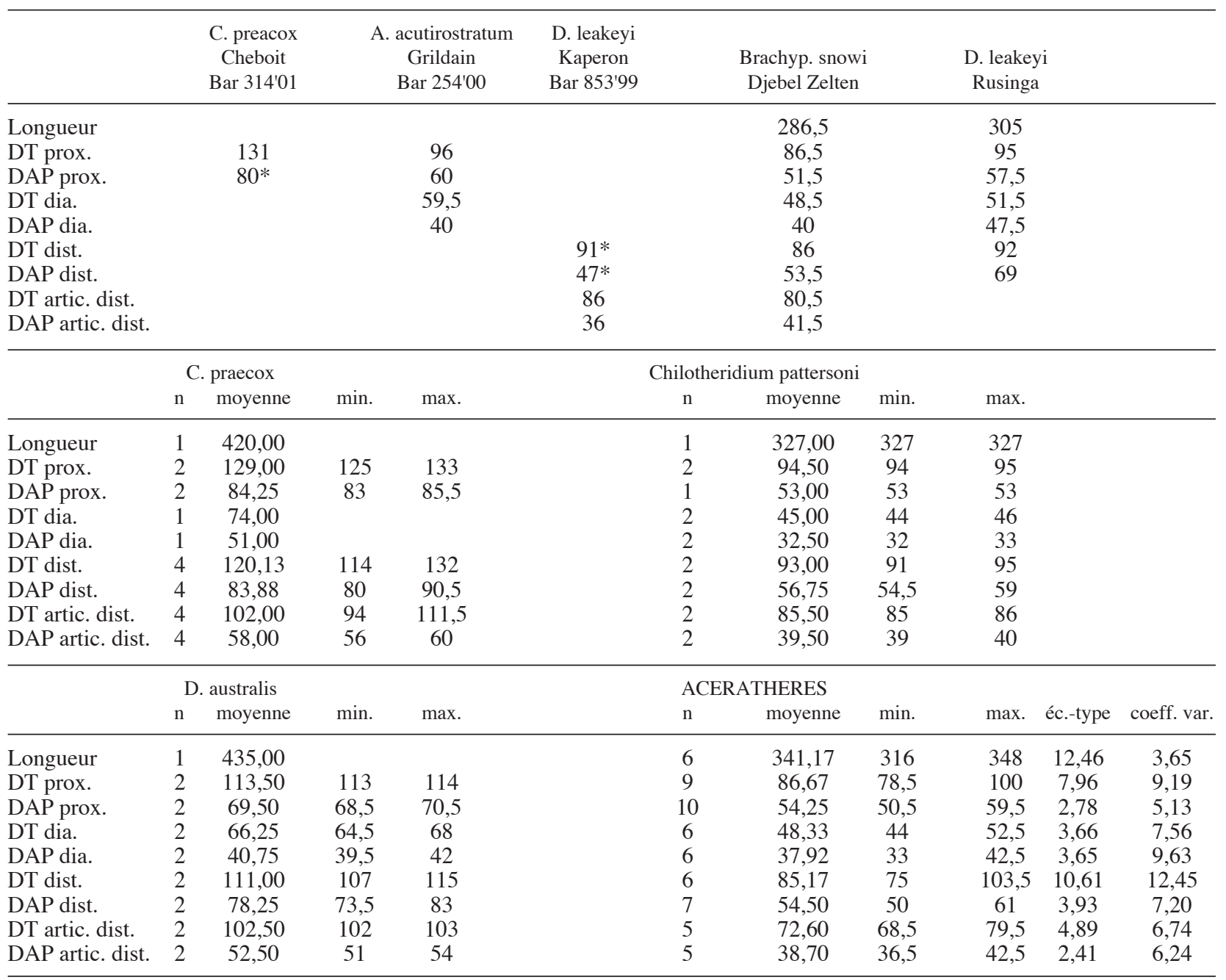

entre dans la marge de variation des D. schleiermacheri du Miocène supérieur européen, la première est sensiblement plus faible que la valeur minimale relevée chez ces derniers.

Les dimensions des jugales supérieures sont données tabl. 3.

La D1/ présente une section horizontale en triangle avec pointe antérieure arrondie; pour les deux spécimens disponibles l'usure est trop forte pour qu'une structure interne soit observable; il existe un cingulum lingual assez faible mais continu; une dépression verticale est présente au milieu de l'ectolophe.

La M3/ est usée et incomplète, il lui manque le tiers médian de l'ectométalophe. Le pli du paracône est fort, il existe un net cingulum labial; le protocône ne montre aucune constriction. L'émail atteint plus de $2 \mathrm{~mm}$ d'épaisseur.

Les I/1 sont aplaties en pelle, avec un bord antérieur asymétrique tranchant; leur usure est nulle; elles ont une racine simple; en vue supérieure la couronne de BAR 1076'02 est pointue en avant, large de $14 \mathrm{~mm}$ pour un diamètre dorso-ventral de $12 \mathrm{~mm}$; BAR 1077 est au contraire élargie $(10 \mathrm{~mm})$ pour un diamètre dorso-ventral de $8 \mathrm{~mm}$.

Les dimensions des jugales inférieures sont données tabl. 5.
La D/1 BAR 1079'02, peu usée, est tranchante en avant et porte une cuspide centrale suivie d'une petite vallée linguale. Le spécimen BAR 6'02, très usé, beaucoup plus étroit que le précédent, montre une couronne à section horizontale en demiellipse pointue en avant.

La D/2 est vierge; elle est nettement trilobée, les lobes étant séparés lingualement et labialement par des synclinaux verticaux; seul le lobe postérieur encadre une véritable vallée linguale. La hauteur atteint 21,5 mm, l'indice d'hypsodontie s'établit à 76,8.

$\mathrm{La} \mathrm{D/3}$, très usée, a ses vallées linguales avec une nette différence de niveau, et ne possède pas de cingulum labial ni lingual.

La M/2, bien usée, présente un synclinal labial faible; les deux vallées linguales ont une forte différence de hauteur; il y a une trace de cingulum labial; l'émail est épais (jusqu'à $3 \mathrm{~mm}$ ).

Les $\mathrm{P} / 2$, toutes deux bien abrasées, sont en triangle très long avec une crête verticale convexe en avant; BAR 1034'99 possède un cingulum labial continu.

La $\mathrm{P} / 3$, peu usée, ne possède aucun cingulum lingual ni labial. Les vallées linguales en $\mathrm{V}$ ont une grande différence de niveau; en vue occlusale le croissant antérieur est à angle droit. 
Mc II

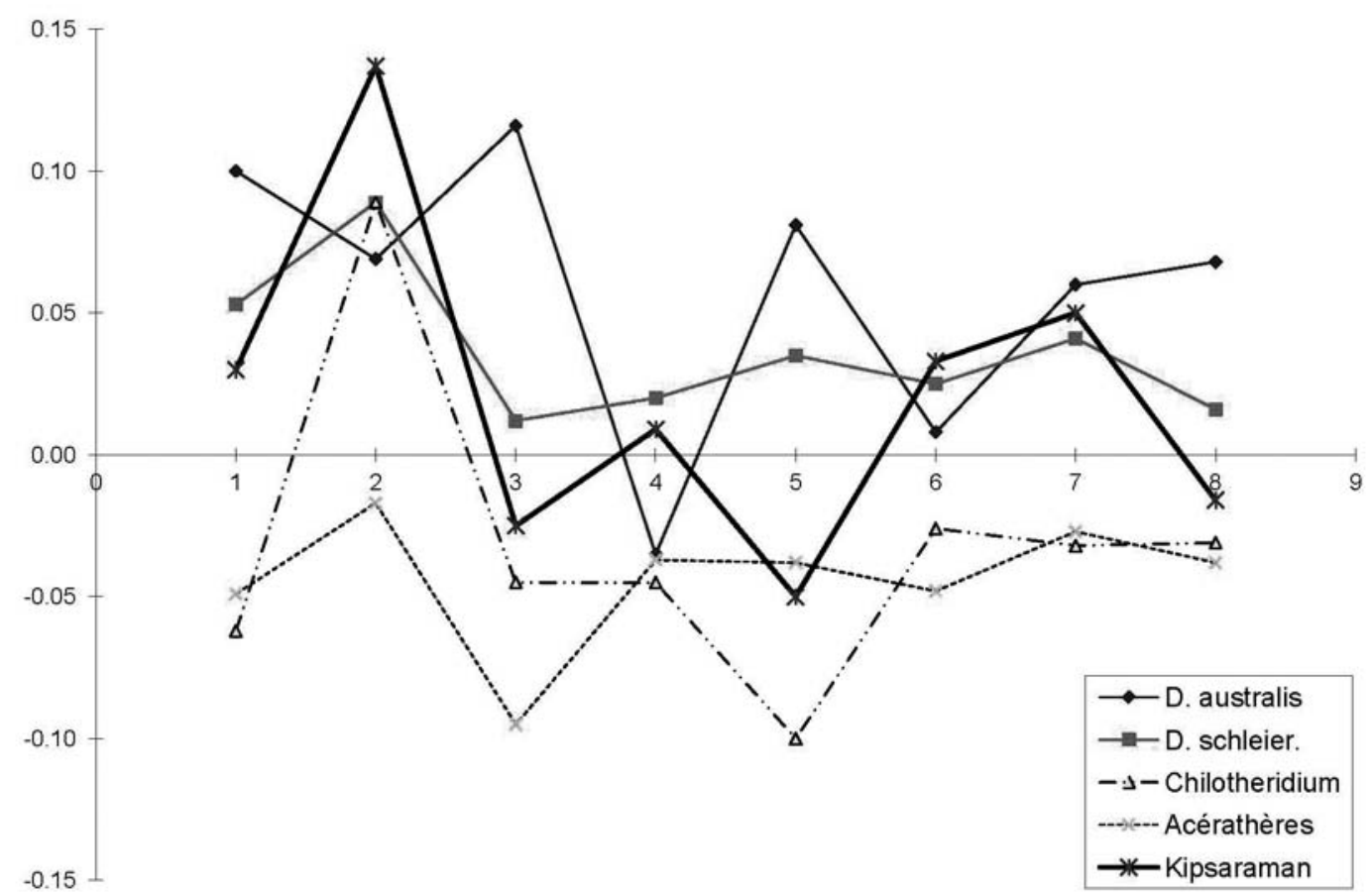

Fig. 2.-Diagramme des rapports des Mc II des Rhinocerotidae néogènes des Tugen Hills (première partie). Le référentiel est Diceros bicornis. Les variables en abscisse sont, dans l'ordre, celles du tabl. 10.

\section{Mc II bis}

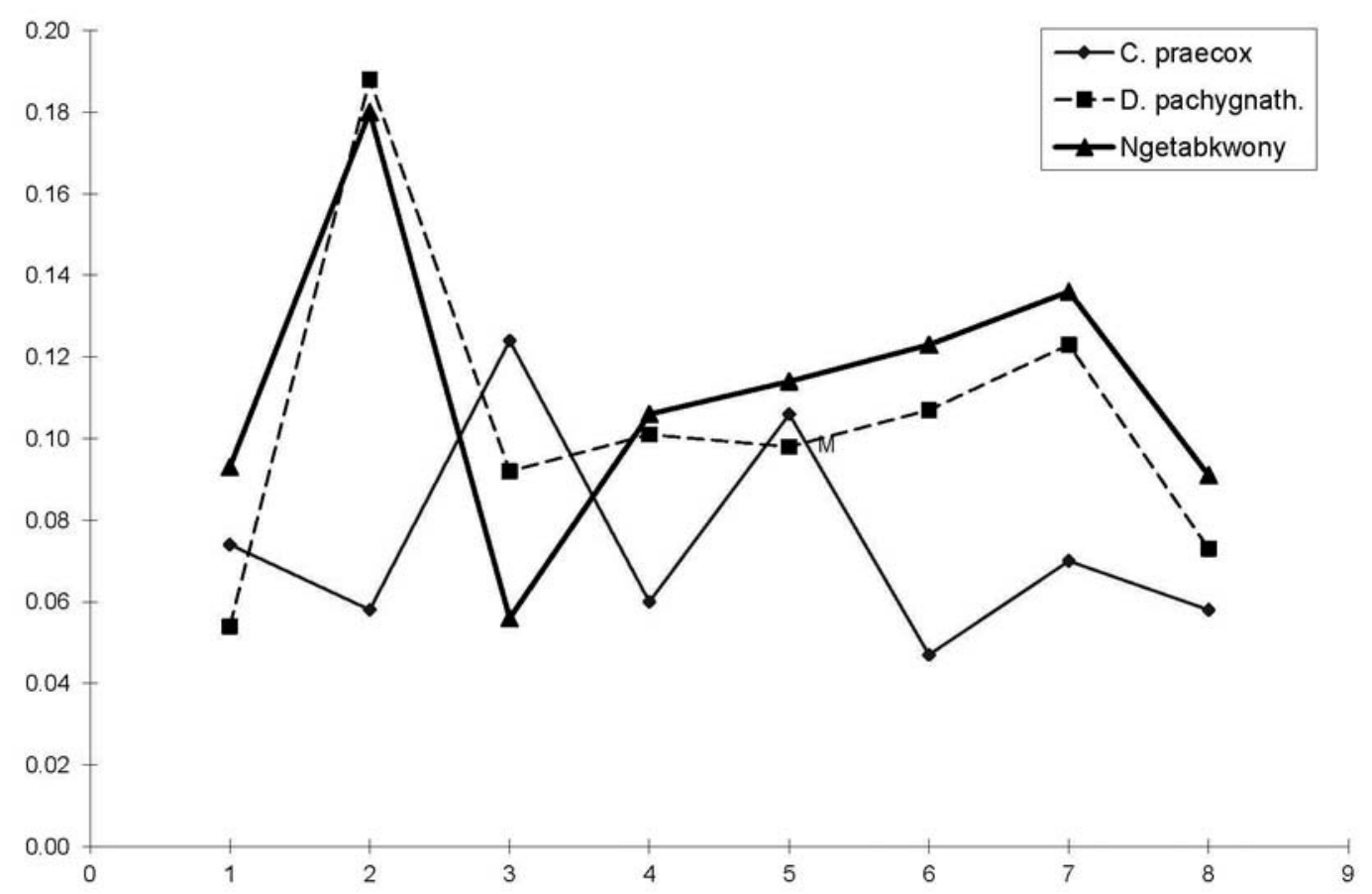

Fig. 3.-Diagramme des rapports des Mc II des Rhinocerotidae néogènes des Tugen Hills (deuxième partie). Le référentiel est Diceros bicornis. Les variables en abscisse sont, dans l'ordre, celles du tabl. 10. 
Les $\mathrm{P} / 4$, qui sont à des stades d'usure très divers, présentent en vue occlusale des croissants à angles droits; il n'y a pas de cingulum labial ni lingual mais le cingulum antérieur déborde labialement. Toutes ont un synclinal labial bien marqué; les vallées linguales en $\mathrm{V}$ présentent une nette différence de niveau.

Le fragment distal de radius de Kaperon est mal conservé et ses diamètres transversal et antéropostérieur distaux ne peuvent être mesurés qu'approximativement; ils correspondent néanmoins à ceux d'un D. leakeyi de Rusinga (tabl. 6).

Le Mc II de Kipsaraman a les dimensions d'un petit $D$. schleiermacheri (tabl. 10), mais les proportions sont un peu différentes (Fig. 2 et 3 ) car l'os est relativement plus large, avec des diamètres transversaux de l'épiphyse proximale, de la diaphyse et de l'épiphyse distale relativement forts. L'articulation proximale (Fig. $1 \mathrm{G}$ ) occupe presque toute la surface supérieure, elle n'est pas en retrait par rapport au bord antérieur de l'os; la tubérosité postérieure de l'épiphyse proximale est très faible. L'articulation proximo-latérale comporte une seule facette, pratiquement rectiligne, avec un dièdre longitudinal séparant la partie répondant au magnum de celle qui répond au Mc III. En face antérieure (Fig. 1 E) l'os apparaît mince et allongé, avec une nette tubérosité latérale à l'extrémité distale de la diaphyse. La face postérieure (Fig. $1 \mathrm{~F}$ ) est modérément creusée dans sa moitié inférieure. La section de la diaphyse est en ellipse plate avec un bord médial à pan coupé oblique.

Affinités: Dicerorhinus leakeyi est un représentant classique des faunes du Miocène moyen d'Afrique orientale, entre 19,5 et $12 \mathrm{Ma}$. Une telle durée de vie montre qu'il a constitué la plus réussie des tentatives de la sous-famille des Dicerorhininae pour prendre pied en Afrique. L'espèce, brachyodonte, était probablement ubiquiste avec une préférence pour les secteurs buissonneux.

\section{Genre Brachypotherium Roger, 1904 Brachypotherium heinzelini Hooijer, 1963}

Le genre Brachypotherium Roger regroupe de gros rhinocéros hippopotamoïdes au corps en barrique et aux pattes courtes mais puissantes. Les I / 2 sont développées en défense; l'ectolophe des jugales supérieures montrent une tendance à l'aplatissement. Les nasaux sont faibles, il n'y avait pas de cornes. Les moeurs plus ou moins amphibies étaient voisines de celles des hippopotames modernes. Le genre est bien connu dans le Néogène d'Eurasie. Trois espèces ont été décrites en Afrique (Guérin, 1980 b, 2000), elles nécessitent une révision (Pickford et al., 1993).

Diagnose spécifique originelle (traduite): Brachypotherium avec P4/ munie d'un cingulum labial, comme B. brachypus, et sans cingulum interne, comme $B$. aurelianense et $B$. snowi. Taille semblable à ces espèces.

Diagnose complétée (d'après les descriptions de Hooijer, 1966): Jugales brachyodontes; les supérieures sont rétrécies en arrière, n'ont pas de nette constriction du protocône et possèdent un anticrochet réduit. Prémolaires supérieures à ectolophe aplati en arrière du pli du paracône. Cingulum lingual bien développé, cingulum externe variable, anticrochet faible ne barrant pas le médisinus. Molaires supérieures à cingulum externe présent ou absent. Jugales inférieures avec synclinal externe aplati, avec ou sans cingulum externe.

Holotype: $\mathrm{P} 4 /$ gauche.
Autre matériel originellement rapporté: divers débris dentaires de Sinda et du Lac Albert, un astragale de Sinda.

Gisement-type: rive droite de la rivière Sinda, en face du ravin d'Ongoliba, Basse Semliki, Congo du NE.

Autres gisements: outre Sinda (plus de $6 \mathrm{Ma})$, B. heinzelini a été signalé à: Arongo Uyoma (Miocene inférieur), Mabaget Formation (anciennement Chemeron Formation-Northern Extension, 5 à 4,5 Ma), Karungu (18 Ma) et Rusinga (18 Ma) au Kénya; Bukwa (entre 17,5 et 18,5 Ma) et Napak II (19,5 Ma) en Ouganda; Karugamania (plus de $7 \mathrm{Ma}$ ) au Congo; Langental (18 Ma) en Namibie.

Gisements des Tugen Hills: Cheparawa (formation Muruyur, 15,5 Ma).

\section{Matériel: M/2 gauche n BAR 637'99 (Cheparawa)}

Description: La M/2, bien usée, possède un synclinal vertical labial très faible, ce qui est une caractéristique du genre; il n'y a pas de cingulum labial. Les dimensions (tabl. 5) sont compatibles avec B. heinzelini.

Affinités: Brachypotherium heinzelini, espèce non retenue par D. Geraads (1989) qui n'indique pourtant pas de synonymie, a vécu en Afrique orientale, centrale et australe entre 19,5 et 4,5 $\mathrm{Ma}$, ce qui constitue une durée énorme. Toutefois sa contemporanéité avec $B$. lewisi à partir de $12 \mathrm{Ma}$ laisse planer un doute sur la détermination spécifique de ses représentants les plus récents.

\section{Brachypotherium lewisi Hooijer \& Patterson, 1972}

C'est la plus récente espèce de brachypothères africains. A l'instar de B. perimense d'Asie du Sud-Est, elle survit dans le Pliocène inférieur.

Diagnose spécifique originelle (traduite): Très grande taille: longueur condylobasale du crâne-type supérieure à $70 \mathrm{~cm}$, largeur antérieure des M1/-M2/ de l'ordre de $90 \mathrm{~mm}$ contre 70 $\mathrm{mm}$ pour $B$. brachypus du Miocène d'Europe et $B$. snowi du Miocène d'Egypte. Nasaux sans corne, minces, pas vraiment longs; le fond de l'échancrure nasale est au dessus de P4/; bord antérieur de l'orbite au dessus de l'avant de M2/; frontaux plats et sans corne; processus inférieurs du squamosal soudés en dessous du pseudo-méat auditif. Incisives supérieures très grosses; jugales supérieures avec ectolophe aplati en arrière du pli du paracône, anticrochet modéré, protocône avec faible constriction, cingulum externe souvent présent. Défenses inférieures (les $\mathrm{I} / 2$, et non pas les canines!) présentes; jugales inférieures brachyodontes à synclinal externe habituellement aplati, cingulum externe souvent développé. Troisième trochanter du fémur fortement développé.

Hooijer \& Patterson (1972) notent à propos d'une molaire supérieure de Ceratotherium praecox découverte à Lothagam parmi des restes de Brachypotherium lewisi que la première se distingue par sa plus forte hypsodontie, son ectolophe très plat ne portant qu'une faible trace de pli du paracône, un net étranglement sur la face antérieure du protocône, l'absence d'anticrochet, le fort crochet, la petite crista et son post-sinus aussi profond que le médisinus.

\section{Holotype: crâne ${ }^{\circ}$ KNM LT 88.}

Autre matériel originellement rapporté: un crâne incomplet écrasé; plusieurs hémimandibules incomplètes; diverses dents isolées; un fémur incomplet. 
Tableau 7.-Dimensions comparées du scaphoïde des Rhinocerotidae néogènes des Tugen Hills

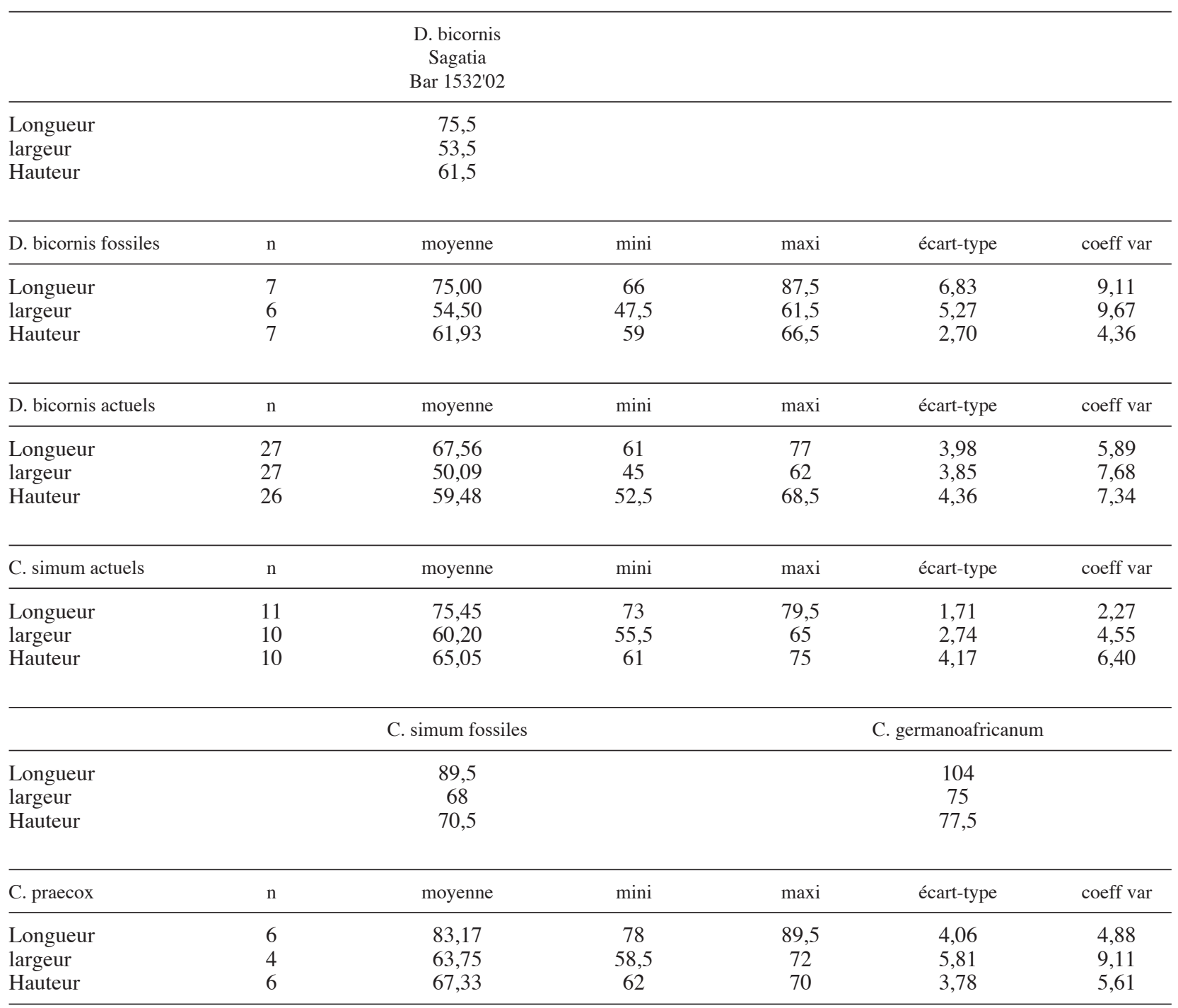

Gisement-type: Lothagam-1, sommet du membre B.

Autres gisements: Lothagam-1 membres B et C (7,2 à 5,5 Ma); Kanapoi (4,5 Ma), Mpesida (6,5 à $6 \mathrm{Ma})$, Ngorora (12 à $11 \mathrm{Ma})$ au Kénya; Sahabi (6,5 Ma) en Libye; Djebel Krechem el Artsouma (10 Ma, Geraads, 1989) en Tunisie.

Gisements des Tugen Hills: Cheboit (formation de Lukeino, $6 \mathrm{Ma}$ ). Kapsomin (formation de Lukeino, 5,8 à 5,7 Ma). Sagatia (formation de Mabaget, 5 à 4,5 Ma).

Matériel: Une D/4 droite n BAR 690'01 (Sagatia); deux fragments de jugales supérieures ${ }^{\circ}$ BAR 239'0 et BAR 496'01 (Cheboit); onciforme gauche (Fig. 1 A-D) n BAR 1902'01 (Kapsomin).

Description: La D/4 de Sagatia, très usée, se caractérise par son synclinal labial très faible, sa partie antérieure plus étroite que la postérieure et son émail épais $(2,5 \mathrm{~mm})$ en dépit de son statut de dent lactéale. Ses dimensions sont données tabl. 5.
Les deux fragments de jugales supérieures de Cheboit présentent une grande taille, un émail épais et un cingulum lingual paraissant continu. Ce matériel n'est malheureusement pas mesurable.

Avec quelques réserves j'attribue à Brachypotherium lewisi l'oncifome de Kapsomin du fait de ses dimensions (tabl. 9) très supérieures et de ses proportions différentes de celles de Ceratotherium praecox: la largeur représente $80,2 \%$ de la longueur absolue, et la hauteur antérieure $62,5 \%$; pour $C$. praecox les valeurs correspondantes sont $74,7 \%$ et $57,5 \%$. La face antérieure au bord distal très bombé est beaucoup plus haute du côté latéral et son angle proximo-latéral est profondément échancré (Fig. 1 A). La face proximale (Fig. 1 C) répond au pyramidal par une grande facette en bandeau, dépourvue de languette postérieure, qui vient au contact de la facette qui, en face latérale, répond au Mc V. La face distale (Fig. $1 \mathrm{~B}$ ), entièrement articulaire, qui répond au magnum, au Mc III et au Mc IV, est trapézoïdale et très élargie. La facette 
Tableau 8.-Dimensions comparées du pyramidal des Rhinocerotidae néogènes des Tugen Hills

\begin{tabular}{|c|c|c|c|c|c|c|}
\hline & & $\begin{array}{c}\text { C. praecox } \\
\text { Cheboit } \\
\text { Bar 496'01 }\end{array}$ & Bar $273^{\prime} 00$ & & $\begin{array}{c}\text { C. praecox } \\
\text { Kinego } \\
\text { Bar } 135^{\prime} 02\end{array}$ & $\begin{array}{c}\text { C. praecox } \\
\text { Sagatia } \\
\text { Bar 594'99 }\end{array}$ \\
\hline $\begin{array}{l}\text { Longueur } \\
\text { largeur } \\
\text { Hauteur }\end{array}$ & & $\begin{array}{l}40 \\
64 \\
56\end{array}$ & $\begin{array}{c}36,5 \\
63 \\
55\end{array}$ & & $\begin{array}{l}40,5 \\
5456,5 \\
5554\end{array}$ & 43 \\
\hline C. praecox & $\mathrm{n}$ & moyenne & $\operatorname{mini}$ & $\operatorname{maxi}$ & écart-type & coeff var \\
\hline $\begin{array}{l}\text { Longueur } \\
\text { largeur } \\
\text { Hauteur }\end{array}$ & $\begin{array}{l}14 \\
15 \\
15\end{array}$ & $\begin{array}{l}48,75 \\
61,70 \\
58,30\end{array}$ & $\begin{array}{c}40,5 \\
57 \\
51\end{array}$ & $\begin{array}{c}57,5 \\
69 \\
69\end{array}$ & $\begin{array}{l}4,77 \\
3,45 \\
4,72\end{array}$ & $\begin{array}{l}9,78 \\
5,60 \\
8,10\end{array}$ \\
\hline $\begin{array}{l}\text { Longueur } \\
\text { largeur } \\
\text { Hauteur }\end{array}$ & $\begin{array}{l}4 \\
3 \\
4\end{array}$ & $\begin{array}{l}47,38 \\
73,33 \\
60,75\end{array}$ & $\begin{array}{c}34,5 \\
72,5 \\
57\end{array}$ & $\begin{array}{c}58,5 \\
74 \\
68,5\end{array}$ & & \\
\hline C. simum actuels & $\mathrm{n}$ & moyenne & $\operatorname{mini}$ & $\operatorname{maxi}$ & écart-type & coeff var \\
\hline $\begin{array}{l}\text { Longueur } \\
\text { largeur } \\
\text { Hauteur }\end{array}$ & $\begin{array}{l}11 \\
11 \\
11\end{array}$ & $\begin{array}{l}47,36 \\
64,59 \\
55,41\end{array}$ & $\begin{array}{c}42 \\
58,5 \\
51,5\end{array}$ & $\begin{array}{c}54 \\
71,5 \\
61\end{array}$ & $\begin{array}{l}3,89 \\
3,47 \\
2,84\end{array}$ & $\begin{array}{l}8,21 \\
5,37 \\
5,12\end{array}$ \\
\hline D. bicornis actuels & $\mathrm{n}$ & moyenne & $\operatorname{mini}$ & $\operatorname{maxi}$ & écart-type & coeff var \\
\hline $\begin{array}{l}\text { Longueur } \\
\text { largeur } \\
\text { Hauteur }\end{array}$ & $\begin{array}{l}25 \\
26 \\
26\end{array}$ & $\begin{array}{l}42,12 \\
55,63 \\
51,21\end{array}$ & $\begin{array}{c}37,5 \\
46,5 \\
41\end{array}$ & $\begin{array}{l}49 \\
61 \\
60\end{array}$ & $\begin{array}{l}3,03 \\
3,57 \\
4,32\end{array}$ & $\begin{array}{l}7,20 \\
6,41 \\
8,44\end{array}$ \\
\hline D. bicornis fossiles & $\mathrm{n}$ & moyenne & $\operatorname{mini}$ & $\operatorname{maxi}$ & & \\
\hline $\begin{array}{l}\text { Longueur } \\
\text { largeur } \\
\text { Hauteur }\end{array}$ & $\begin{array}{l}3 \\
3 \\
3\end{array}$ & $\begin{array}{l}43,50 \\
56,83 \\
50,67\end{array}$ & $\begin{array}{c}42 \\
56 \\
47,5\end{array}$ & $\begin{array}{c}45 \\
57,5 \\
53\end{array}$ & & \\
\hline
\end{tabular}

articulaire médiale, qui répond au semi-lunaire, est en triangle arrondi (Fig.1 D).

Affinités: Brachypotherium lewisi a vécu au Miocène supérieur et au Pliocène ancien, entre 12,5 et 4,5 Ma, en Afrique du Nord et en Afrique orientale. La superposition partielle de son extension temporelle avec celle de $B$. heinzelini paraît peu plausible et nécessite une révision du matériel correspondant. Le mode de vie de ces brachypothères était aquaphile, assez proche de celui des Hippopotames.

\section{Genre Chilotheridium Hooijer, 1971 Chilotheridium pattersoni Hooijer, 1971}

La sous-famille des Chilotheriinae montre des convergences avec la sous-famille précédente: il s'agit la aussi de rhinocéros hippopotamoïdes au corps en barrique et à pattes courtes. Toutefois la taille générale est petite, il existait une petite corne dans les deux sexes, les I /2 sont faibles, les jugales sont hypsodontes et la main est tétradactyle. Le genre principal Chilotherium, abondant dans le Néogène d'Europe orientale et d'Asie, est absent d'Afrique où il est remplacé par le genre endémique Chilotheridium Hooijer.

Diagnose originelle (traduite): elle est commune au genre et à l'espèce. Une seule corne nasale, petite, dans les deux sexes. Prémaxillaires faibles, pas d'incisives supérieures. Frontaux et pariétaux pneumatisés; orbite plus basse que chez Chilotherium; crâne et occiput plutôt étroits; crêtes pariétales peu éloignées; processus inférieurs du squamosal non fusionnés en dessous du pseudo-méat auditif. Partie symphysaire de la mandibule étroite, légèrement élargie vers l'avant. Jugales bien hypsodontes comme chez Chilotherium, et construites de la même façon: les supérieures ont un pli du paracône qui s'évanouit à proximité du collet, et un ectolophe aplati postérieurement; protocône bien décalé par son étranglement et aplati 
Tableau 9.-Dimensions comparées de l'onciforme des Rhinocerotidae néogènes des Tugen Hills

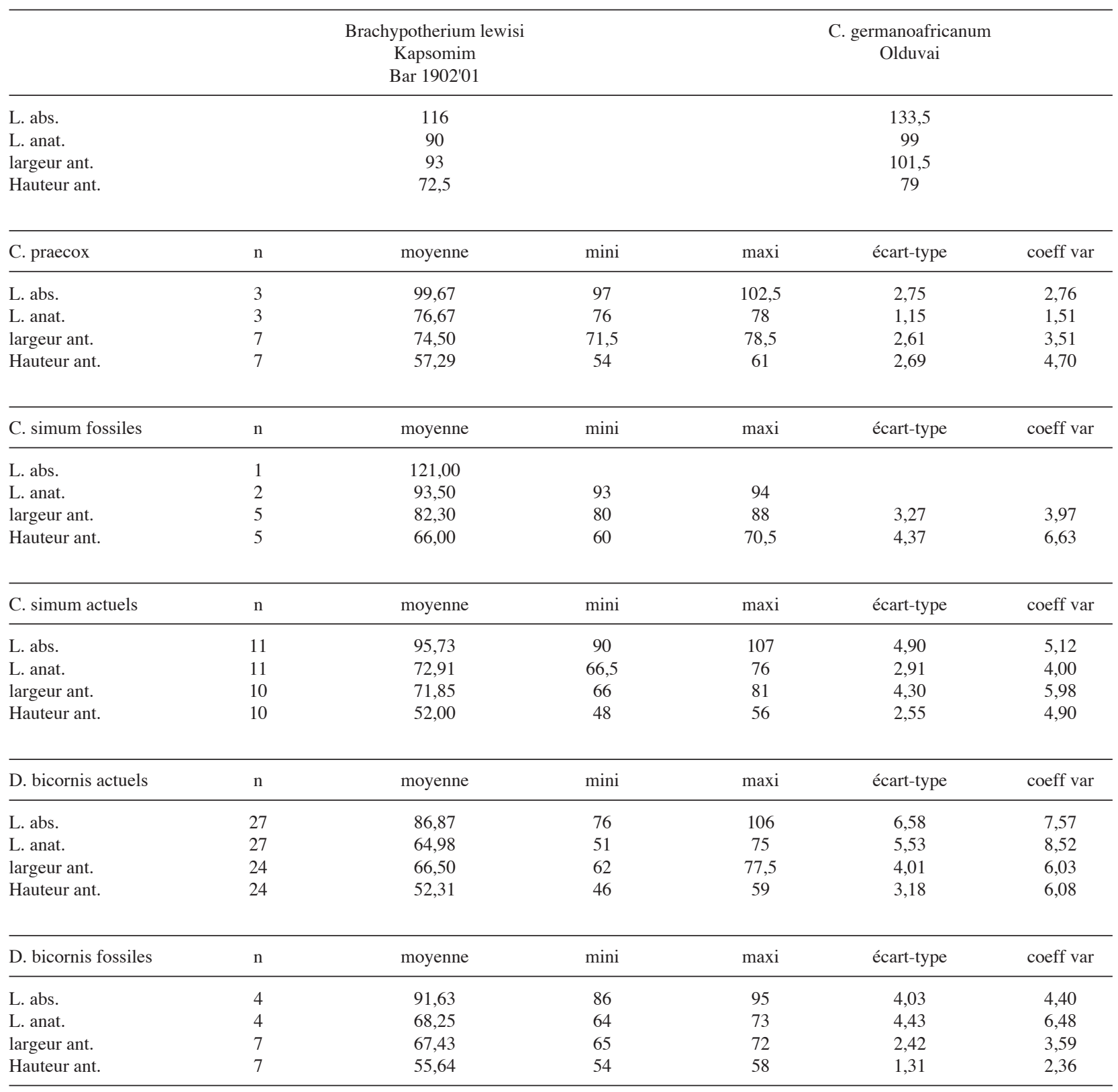

intérieurement; étranglement antérieur sur le métalophe, soulignant l'hypocône; anticrochet proéminent à la base, se recourbant vers l'intérieur à l'entrée du médisinus; crochet normalement bien développé, crista faible ou absente; aux M3/ le métacône est dilaté à sa base; cingulum antérieur fort, cingulum interne faible et formant habituellement un tubercule à l'ouverture de la vallée interne. Défense inférieure à section subtriangulaire, déprimée dorso-ventralement; bord interne aiguisé par l'usure, bord inféro-externe arrondi, bord supéroexterne avec côte. Omoplate basse et large. Os des membres peu raccourcis, ceux des deuxième segments ne sont pas ankylosés. Radius avec facette pour le pyramidal. Semi-lunaire sans facette répondant au cubitus. Métacarpien V fonctionnel, atteignant les trois quarts de la longueur du Mc IV; métapodes latéraux quelque peu divergents vers l'arrière. Fémur avec troisième trochanter petit. Calcanéum dépourvu de facette tibiale. Naviculaire presque rectangulaire, cuboïde plus large que haut. Mt III avec petite facette pour le cuboïde.

Holotype: crâne n 70-64K, B12, de Loperot.

Autre matériel originellement rapporté: Hooijer (1971) décrit un vaste hypodigme comprenant plusieurs spécimens de presque tous les éléments du squelette. 
Gisement-type: Turkana Grit près de Loperot, Turkana District, Kénya.

Autres gisements: outre Loperot (17 Ma), Ch. pattersoni est connu de Kirimum (15 Ma), Ngorora (12 à $11 \mathrm{Ma})$, Ombo (16 $\mathrm{Ma}$ ) et Rusinga (18 Ma), tous au Kénya; Bukwa (environ 18 Ma) en Ouganda (Hooijer, 1973); Arrisdrift (17 Ma) et Grillental (21-20 Ma) en Namibie (Guérin, 2000).

Gisements des Tugen Hills: Ainopchorit (formation de Ngerngerwa, 9,5 Ma)

Matériel: P3/ droite n BAR 27'98.

Description: Cette dent très petite (tabl. 3), d'usure moyenne, dont l'angle antéro-labial est brisé, ce qui empêche l'observation du pli du paracône, se caractérise par sa forte hypsodontie et son ectolophe bombé. Il n'y a pas d'étranglement du protocône. Crista et crochet sont présents. Il n'y a pas de cingulum lingual ni labial.

Affinités: C'est surtout du fait de ses dimensions et de son hypsodontie que j'attribue à $C h$. pattersoni la prémolaire supérieure d'Ainopchorit. Elle constitue le plus récent témoignage de l'espèce, connue en Afrique orientale et australe et dont la durée de vie était jusqu'ici estimée entre 21 et $11 \mathrm{Ma}$. L'espèce était très probablement aquaphile et vivait en environnement fluviatile ou palustre.

\section{Genre Aceratherium Kaup, 1832 Aceratherium acutirostratum (Deraniyagala, 1951)}

L'espèce, originellement attribuée à un genre particulier, Turkanatherium, a été rapportée au genre Aceratherium Kaup par C. Arambourg (1959) et réétudiée par D.A. Hooijer (1966). Aceratherium représente le principal sous-ensemble de la sousfamille des Aceratheriinae. Largement répandu dans le Néogène d'Eurasie, il regroupe des rhinocéros tapiroïdes de taille moyenne à grande, sans corne, aux os nasaux courts et grêles; les I/2 sont généralement développées en une puissante paire de défenses; les jugales sont très brachyodontes, les supérieures ont un ectolophe plus ou moins plat. La main est tétradactyle. Deux espèces sont connues en Afrique (Guérin, 1980 b, 2000). A.W. Gentry (1987), se fondant sur leur profil crânien concave et la grande taille de leurs jugales supérieures, a émis quelques doutes sur l'appartenance réelle de ces espèces au genre Aceratherium, mais je ne le suivrai pas car le genre est relativement polymorphe.

Diagnose spécifique: remaniée informellement par D.A. Hooijer (1963, 1966 et 1968 a): nasaux allongés, faibles; double crête sagittale faible. Pas de corne. Prémaxillaires bien développés et plus étendus vers l'avant que les nasaux. Occiput élevé, donnant un profil fronto-pariétal concave. Fond de l'échancrure nasale au dessus du milieu de M3/. Prémolaires supérieures à anticrochet fermant le médisinus et fort cingulum lingual. Molaires supérieures avec fort anticrochet et nette constriction du protocône. Défenses aplaties dorso-ventralement, à section ovale, courbées vers l'extérieur, leurs convexités respectives étant opposées. lan).

Holotype: un crâne, conservé au Musée de Colombo (Cey-

Gisement-type: Moruaret Hill (ou Moruorot) près de Losodok (ou Lothidok), Turkana district, Kénya.
Autres gisements: outre Moruaret Hill (16,2 Ma), A. acutirostratum est signalé à Alengerr Beds (14 à $12 \mathrm{Ma}$ ), Mabaget Formation (5 à 4,5 Ma), Karungu (18 Ma), Ngorora Formation (sommet du Miocène moyen), Ombo (18 Ma), Rusinga (18 Ma) au Kénya; Napak I (19,5 Ma) en Ouganda; Karugamania (plus vieux que $7 \mathrm{Ma}$ ) et Sinda (plus de $6 \mathrm{Ma}$ ) au Congo. L'espèce vient aussi d'être reconnue à Fiskus (Sperrgebiet septentrional, 19 à 20 Ma) en Namibie (Guérin, 2008).

Gisements des Tugen Hills: Cheparawa (formation Muruyur, 15,5 Ma). Kipsaraman (formation Muruyur, 15,5 - $15 \mathrm{Ma}$ ). Grildain (formation Ngorora, $13 \mathrm{Ma}$ ). Kabarsero (formation Ngorora, 12,5 Ma).

Matériel: un crâne adulte n BAR 305'99 (Grildain); un crâne juvénile avec ses rangées dentaires lactéales $n^{\circ}$ BAR 665'99 (Cheparawa); mandibule juvénile n BAR 304'99 (Grildain) avec ses rangées dentaires incomplètes; M1/ gauche isolée ${ }^{\circ}$ BAR 953’02 (Kabarsero); deux jugales supérieures isolées $n^{\circ}$ BAR 236'02 et 1072'02 (Kipsaraman); les deux tiers proximaux d'un radius gauche n BAR 254'00 (Grildain).

Description: Le crâne adulte de Grildain n'est pas déformé mais très mal conservé, sa face droite est complètement érodée, très peu de ses dimensions sont mesurables. Il ne porte qu'une seule dent, la M3/ gauche très usée et non mesurable. Les dimensions (tabl. 2), notamment la largeur de l'arrière-crâne au niveau de la constriction post-orbitaire, sont du même ordre de grandeur que pour Aceratherium tetradactylum et A. incisivum. La distance du fond de l'échancrure nasale à l'orbite est plus forte que pour les deux espèces européennes alors que la longueur de l'échancrure nasale est plus faible. Le profil crânien n'est finalement pas très différent.

Le crâne juvénile de Cheparawa est déformé obliquement mais presque complet, il est étudiable sur son côté droit. Les nasaux se terminent vers l'avant en pointe très étroite $(17,5$ $\mathrm{mm}$ ); l'échancrure nasale s'étend sur une longueur de $82 \mathrm{~mm}$ et son fond se situe au dessus de l'avant de D2/; la distance de ce fond à l'orbite est d'environ $60 \mathrm{~mm}$. L'avant de l'orbite est au dessus de l'intervalle D3/-D4/. Il existe un net processus postorbitaire, mais pas de processus sus-orbitaire, et peut-être pas non plus de processus anté-orbitaire. La distance de la pointe du nasal à la protubérance occipitale externe est de $380 \mathrm{~mm}$.

La mandibule juvénile de Grildain n'a conservé que ses premières dents de lait; la symphyse est en bon état sauf pour son angle antérieur gauche, et l'on n'observe pas d'alvéole pour les incisives. L'arrière de la symphyse se situe sur l'avant des D/2.

La rangée dentaire lactéale supérieure de Cheparawa est complète à droite (Fig. 4 A), sa longueur est 161,5 mm, dont 101,5 mm pour le segment D3/-D4/. Les dimensions des molaires de lait sont données tabl. 4. La D1/, moyennement usée, possède un ectolophe régulièrement convexe et ne montre aucun pli externe; il n'y a pas de protolophe; il existe une médifossette fermée, et un cingulum lingual continu. La D2/ est bien usée; l'ectolophe régulièrement convexe porte en son milieu un pli petit mais large; la dent est nettement plus large au niveau du protolophe qu'à celui du métalophe; le cingulum lingual est continu; pas d'étranglement du protocône; aucun pli interne n'est visible. La D3/ moyennement usée possède un fort pli du paracône et un métastyle saillant vers l'extérieur; un cingulum lingual discontinu barre l'embouchure de la vallée interne; le protocône est nettement étranglé sur ses deux faces. La D4/ en dehors de sa taille ne se distingue de la D3/ que par son pli du paracône plus fort. 

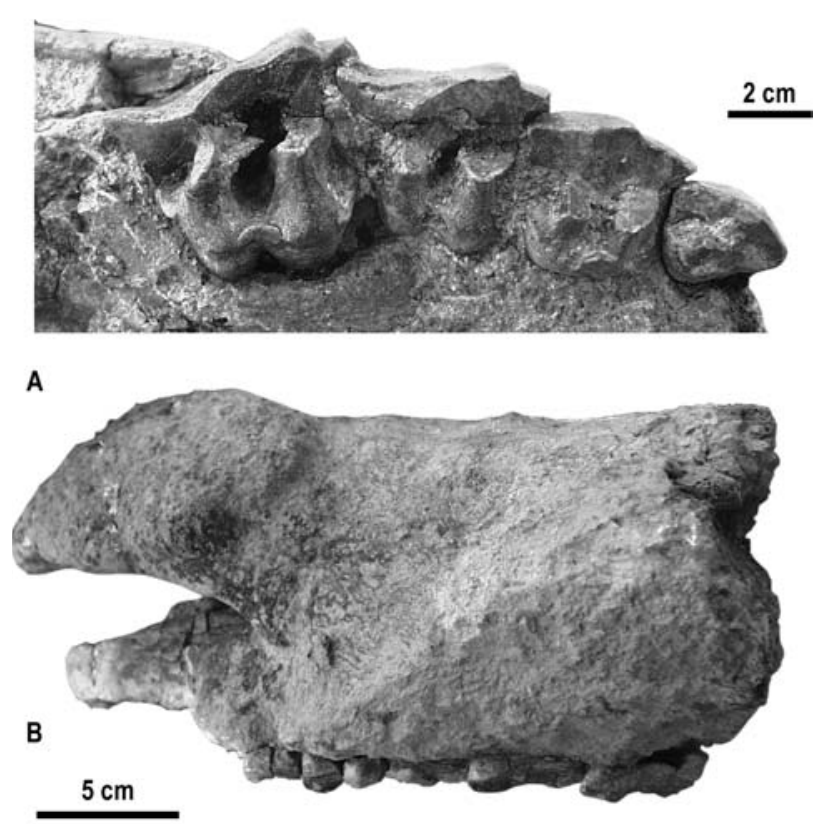

Fig. 4.-Aceratherium acutirostratum et Paradiceros mukirii. A: A. acutirostratum de Cheparawa, crâne juvénile n' BAR 665'99, rangée dentaire supérieure droite, vue occlusale. B: $P$. mukirii de Cheparawa, crâne n BAR 215'99, vue latérale gauche.

La M1/ de Kabarsero, bien usée, possède un émail épais (plus de $2 \mathrm{~mm}$ ), un ectolophe avec un pli du paracône épais et peu détaché; le protocône est étranglé sur ses deux faces. Le seul repli interne est un fort crochet. Un cingulum s'étend sur le bord lingual du protocône et vient barrer l'entrée du médisinus. Les dimensions sont données tabl. 4.

Deux M2/ ou 1/ de Kipsaraman, non mesurables car dépourvues de leur angle antéro-labial, ont un cachet acérathéroïde par leur brachyodontie, leur net crochet et le fort étranglement de leur protocône. L'une possède un cingulum lingual continu.

Trois molaires de lait sont portées par la mandibule de Grildain, leurs dimensions sont données tabl. 5. D/1 a une section elliptique pointue en avant; elle n'est pas usée et sa hauteur est $16,5 \mathrm{~mm}$, l'indice d'hypsodontie s'établissant à 110 . La D/2 droite, bien usée, porte une fossette fermée à l'arrière de sa face occlusale.

Le radius de Grildain montre une face articulaire proximale dont la facette externe, bien étendue latéralement, est en net retrait par rapport à la facette médiale; le bord antérieur de la face est très peu déprimé au niveau du contact entre les deux facettes. Les dimensions sont données tabl. 6, elles sont proches des valeurs maximales relevées chez les A. tetradactylum et $A$. incisivum européens, et la morphologie de l'épiphyse proximale est plus proche de la deuxième espèce que de la première.

Affinités: A. acutirostratum a vécu en Afrique orientale et centrale pendant un intervalle de temps considérable, de 19,5 à 4,5 Ma, soit une quinzaine de millions d'années si sa présence à Chemeron Formation-Northern extension est avérée; dans le cas contraire la durée de vie de l'espèce serait sensiblement plus courte.

Bien qu'un peu plus grand, il rappelle par son anatomie, ses dimensions et ses proportions les A. tetradactylum et A. incisi- vum du Miocène moyen et supérieur d'Europe occidentale. Il avait très probablement le même mode de vie, assez proche de celui des tapirs actuels, affectionnant les bordures de corps d'eau et les zones marécageuses.

\section{Genre Paradiceros Hooijer, 1968 Paradiceros mukirii Hooijer, 1968}

Cette petite forme du Miocène moyen appartient à la sousfamille des Dicerotinae, qui inclut les deux espèces africaines actuelles Diceros bicornis (le rhinocéros «noir») et Ceratotherium simum (le rhinocéros «blanc»). La sous-famille se définit par la possession de deux cornes, une face courte, une symphyse mandibulaire brève, l'absence de dents antérieures fonctionnelles et, sauf pour Ceratotherium dont la denture très hypsodonte est particulièrement évoluée, des jugales brachyodontes, les supérieures ne comportant qu'un seul pli bien marqué sur leur ectolophe, le pli du paracône.

Le genre Diceros apparaît avec D. australis Guérin, 2000, du Miocène moyen ancien de Namibie, cursorial, de grande taille, et doté d'incisives inférieures vestigiales. Après un important hiatus, il est attesté à nouveau au Miocène supérieur par un groupe de grandes formes dont l'extension est périméditerranéenne. Les premiers Ceratotherium datent de la fin du Miocène. Le genre Paradiceros Hooijer, 1968, de petite taille, du Miocène moyen récent d'Afrique orientale et du Maghreb, apparaît quant à lui comme un rameau latéral sans descendance.

Diagnose générique (traduite): Deux cornes, respectivement nasale et frontale. Processus inférieurs du squamosal non fusionnés. Occiput vertical. Symphyse mandibulaire raccourcie mais pas élargie, édentée chez l'adulte. Dents jugales brachyodontes à protocône étranglé et anticrochet proéminent. Dernière molaire supérieure subtriangulaire. Molaires supérieures avec vallées à débouché large et bas, plus haut pour les prémolaires supérieures. Membres et certains os du pied plus courts que chez Aceratherium et Dicerorhinus, mais pas aussi raccourcis que chez Brachypotherium ou Chilotherium.

Diagnose spécifique (traduite): Echancrure nasale peu profonde, étendue jusqu'au niveau de P2/. D2/ avec mésostyle. Molaires de lait et molaires antérieures à protocône étranglé et anticrochet saillant, c'est moins net aux arrière-molaires et aux prémolaires. Os des membres modérément raccourcis. Astragale non surbaissé.

Holotype: un crâne juvénile avec ses dents de lait, conservé au Musée de Nairobi. Un bon moulage se trouve au Natural History Museum de Londres, où j'ai pu l'étudier.

Autre matériel originellement rapporté: deux fragments de maxillaires juvéniles avec molaires de lait, deux molaires de lait isolées; une mandibule juvénile avec ses molaires de lait; une molaire de lait inférieure isolée; une M1/ vierge isolée; deux molaires supérieures usées isolées; deux prémolaires supérieures isolées; un crâne écrasé avec denture complète; un fragment de crâne avec le segment prémolaire gauche; une mandibule adulte; un fragment d'hémimandibule déformée; un atlas; un humérus complet; un fragment distal de radius; un cubitus; un magnum; un métacarpien III complet; deux fémurs et un tibia mal conservés; cinq astragales; trois calcanéums; un cuboïde; un Mt II complet; deux Mt III complets; les trois phalanges d'un doigt abaxial.

Gisement-type: Fort Ternan (Kénya, 13,7 Ma) 

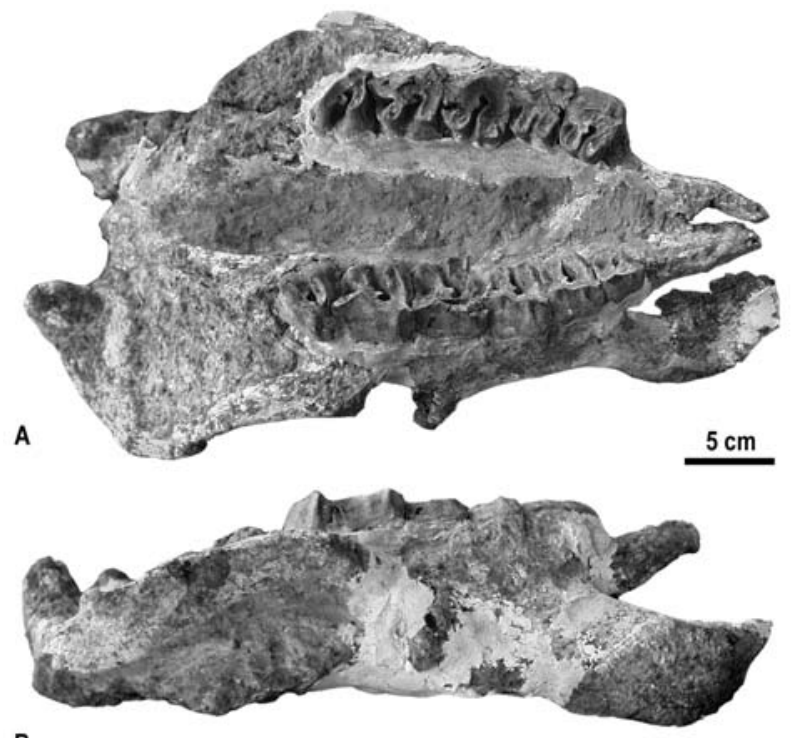

B

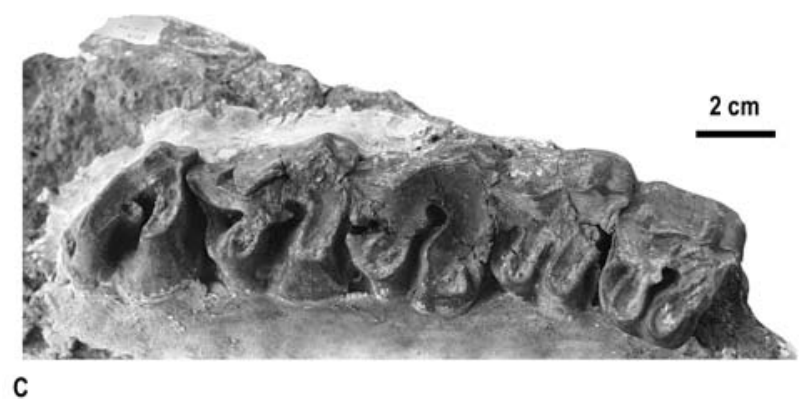

Fig. 5.-Paradiceros mukirii de Cheparawa, crâne $n^{\circ}$ BAR 214'99. A: vue inférieure. B: vue latérale gauche. C: rangée dentaire supérieure droite, vue occlusale.

Autres gisements: peut-être Maralal, également au Kénya (Hooijer, 1968). L'espèce est présente aussi à Kisegi (13 à 14 Ma) en Ouganda (Guérin, 1994), et à Beni Mellal (12,5 Ma) au Maroc (Guérin, 1976).

Gisements des Tugen Hills: Cheparawa (formation Muruyur, $15,5 \mathrm{Ma})$

Matériel: crâne facial BAR 215'99 (Cheparawa); crâne BAR 214'99 (Cheparawa)

Description:Crânes (Fig. 4 B et 5 A-B): l'un est très incomplet, l'autre écrasé. Les dimensions sont données tabl. 2.

Les nasaux sont très élargis en avant, fortement convexes; leur surface d'insertion pour la corne nasale est très irrégulière, en chou-fleur, avec une partie centrale plus lisse, déprimée, étendue sur un peu plus de $30 \mathrm{~mm}$ de large. Sur le spécimen $\mathrm{n}^{\circ}$ BAR 214'99, l'épaisseur de l'os dépasse $40 \mathrm{~mm}$ au maximum de sa convexité, qui se situe à plus de $60 \mathrm{~mm}$ de l'extrémité rostrale. Le fond de l'échancrure nasale est au dessus de l'arrière de P2/ (BAR 214'99) ou de l'avant de P 3/ (BAR 215'99).

Le bord antérieur de l'orbite, qui est à plus de $215 \mathrm{~mm}$ de la pointe du nasal, se situe au dessus de l'arrière de $\mathrm{M} 1$.
Les intermaxillaires sont longs (du côté gauche du crâne ${ }^{\circ}$ BAR $215^{\prime} 99$, il y a près de $80 \mathrm{~mm}$ entre l'avant et la première jugale -P2/- du même côté), non jointifs rostralement, et dépourvus d'alvéole dentaire.

L'échancrure pré-palatine n'atteint pas le niveau des premières jugales.

L'arcade zygomatique, déprimée sur l'essentiel de sa longueur, atteint son maximum de largeur dans sa partie postérieure; son gond articulaire, conservé du côté gauche du $n^{\circ}$ BAR 214'99, s'étend transversalement sur 95 mm.

Dents jugales supérieures: seules celles du crâne $n^{\circ}$ BAR 214'99 sont étudiables (Fig. 5 A et C). Leurs dimensions sont données tabl. 4.

P2/: l'usure est forte, elle a fait confluer le protocône et l'hypocône, créant ainsi une médifossette fermée alors que la dent ne possède ni crista, ni crochet. L'ectolophe régulièrement convexe porte un très petit pli du paracône. Il existe un cingulum lingual continu, ainsi qu'un cingulum postérieur limitant une post-fossette très nette dont il est séparé par un glacis.

P3/: l'usure est moyenne. L'ectolophe sub-plat est muni d'un pli du paracône s'ennoyant à $10 \mathrm{~mm}$ du collet, et d'un pli du métacône à peine discernable. Il n'y a pas d'étranglement du protocône. Le seul repli interne est un petit crochet. On observe un cingulum interne très net mais interrompu sur la face linguale du protocône; un cingulum postérieur très fort limite une petite post-fossette dont il est séparé par un glacis.

$\mathrm{P} 4$ /: la morphologie est très semblable à celle des $\mathrm{P} 3 /$, mais le cingulum lingual est moins marqué.

M1/: l'usure est moyenne. L'ectolophe est sub-plat avec un pli du paracône très faible et disparaissant loin du collet, mais le profil longitudinal se rebrousse vers l'arrière au niveau du métastyle. Le seul repli interne est un petit crochet. Le protocône n'est pas étranglé, son bord lingual est carré. On observe un début d'étranglement sur la face antérieure de l'hypocône. Il n' y a pas de cingulum lingual; en revanche, comme pour les prémolaires, un puissant cingulum postérieur induit une post-fossette bien marquée.

M2/: sa morphologie est voisine de celle de la molaire précédente, sauf pour trois points: le protocône subit un début d'étranglement; la vallée interne est plus ouverte, avec un profil transversal en $\mathrm{U}$; la post-fossette est profonde.

M3/: l'usure est faible. Comme pour les autres molaires le seul repli interne est un crochet. Le pli du paracône est plus fort. Il n'existe plus qu'une trace de cingulum lingual, au débouché de la vallée interne. Un court bourrelet se trouve sur l'extrémité postérieure de l'ectométalophe.

Affinités: La description originelle de D.A. Hooijer (1968) est assez complète. Le matériel de Cheparawa permet toutefois de la préciser sur certains points:

- le nasal est remarquablement large, très épais, et sa face supérieure est très irrégulière;

- les intermaxillaires sont longs, et il n'y a pas d'incisives supérieures:

- les prémolaires ne possèdent ni étranglement du protocône ni anticrochet, il faudra donc retirer ces deux caractères de la diagnose.

$P$. mukirii présente de nombreuses analogies morphologiques avec Diceros bicornis et devait avoir un milieu de vie assez semblable. Cheparawa est le plus ancien gisement de l'espèce, dont la durée de vie peut désormais être estimée entre 15,5 et $12 \mathrm{Ma}$. 


\section{Genre Certotherium Gray 1868 Ceratotherium praecox Hooijer \& Patterson, 1972}

Il s'agit de la plus ancienne espèce du genre, qui marque d'emblée une très nette évolution crâniale et dentaire vers l'hypsodontie et l'adaptation aux biotopes très ouverts; $C$. praecox annonce l'énorme $C$. germanoafricanum (Hilzheimer, 1925) du Pliocène terminal/Pléistocène ancien d'Afrique orientale, le C. mauritanicum (Pomel, 1895) du Plio-Pléistocène du Maghreb et l'actuel C. simum (Burchell, 1817) apparu au Pléistocène moyen (Guérin, 1979). Une stricte application du Code international de nomenclature zoologique obligerait à le désigner C. efficax (Dietrich, 1942), ce que je refuse de faire au nom du principe de la stabilité de la nomenclature.

D. Geraads (2005) a cru reconnaître l'espèce maghrébine Ceratotherium mauritanicum dans le Pliocène d'Afrique orientale (Hadar dans la vallée de l'Aouache en Ethiopie, Laetoli en Tanzanie, Est- et Ouest-Turkana et Rawi au Kénya) ainsi qu'en Afrique du Sud (Langebaanweg). Nous connaissons bien l'essentiel de ce matériel (Guérin, 1987, 1994) et il s'agit en fait dans tous les cas de Ceratotherium praecox, dont D. Geraads reconnaît certes l'existence à Hadar, mais qu'il place sans argument convaincant dans le genre Diceros et qu'il confond d'ailleurs avec Diceros bicornis aussi bien à Hadar qu'à Lothagam et Laetoli. Nous estimons que la confusion des deux genres n'est pas justifiée, et celle des espèces encore moins, tous ces taxons se différenciant très clairement les uns des autres par la morphologie, les dimensions et les proportions du crâne, des dents et du squelette des membres. De notre point de vue $C$. mauritanicum n'a jamais été pour l'instant attesté hors d'Afrique du Nord, il est issu de C. praecox, au même titre que $C$. germanoafricanum et $C$. simum et n'a pas de descendance; enfin c'est le genre évolué Ceratotherium qui est issu du genre plus primitif et peu spécialisé Diceros, et non l'inverse!

Diagnose spécifique originelle (traduite): Crâne se distinguant de celui de $C$. simum par la plus grande concavité du toît crânien, le crâne moins étendu postérieurement, l'occiput plus vertical. Jugales moins hypsodontes, lophes et lophides pas particulièrement obliques; angle antéro-interne des dents supérieures non arrondi; pas de médifossette aux P4/-M2/, pas de fossettide aux jugales inférieures; cingulum interne variable aux jugales supérieures.

Holotype: crâne incomplet $n^{\circ}$ KNM KP 36 de Kanapoi (Kénya, SE du District Turkana).

Autre matériel originellement rapporté: fragments de crânes, fragments de mandibules, dents isolées, humérus incomplet.

Gisement-type: Kanapoi (Kénya).

Autres gisements: Hooijer \& Patterson (1972) indiquent, outre Kanapoi (4,5 Ma), Lothagam-1 membres B et C (7,2 à 5,5 $\mathrm{Ma}$ ) et Ekora (4 à 3,5 Ma), aussi au Kénya; dans le même pays l'espèce est attestée des Aterir Beds (4 Ma), de la Chemeron Formation loc. JM 507, de Koobi Fora (Kubi Algi, 4 à 2,5 Ma), de Lukeino (6 à 5,7 Ma), de Mpesida (6,5 à $6 \mathrm{Ma})$. C. praecox est également connu en Ethiopie à l'Omo (Mursi et base de Shungura, de plus de 4 à $3 \mathrm{Ma}$ ), de Hadar (Sidi Hakoma, 3,4 à 3,1 Ma). Il est signalé à Laetoli (3,8 à 3,5 Ma) en Tanzanie et dans les formations de Nkondo (5 Ma), Warwire (4,5 Ma) et Hohwa en Ouganda (2,3 Ma) (Hooijer \& Patterson, 1972; Guérin, 1979, 1980 b, 1985, 1987 a, 1994). L'espèce est également présente en Afrique du Sud à Langebaanweg-E quarry (5 à 4
Ma), Limeworks Cave-Makapansgat (3,4 à $3 \mathrm{Ma})$ et à Swartlintjes Farm, Hondeklipbaai (Namaqualand, Cape Province). Enfin A. Likius (2002) la signale dans le Pliocène du Tchad à Kollé (5 à $4 \mathrm{Ma}$ ) et Koro Toro (3,5 à $3 \mathrm{Ma})$.

Gisements des Tugen Hills: Cheboit (formation de Lukeino, $6 \mathrm{Ma}$ ). Kapcharar (formation de Kapairaina, 5,2 Ma). Kapcheberek (formation de Lukeino, 5,8 Ma). Kapchebrit (formation de Mabaget, 5 à 4,5 Ma). Kapchepkweren (formation de Lukeino, $6 \mathrm{Ma}$ ). Kapsomin (formation de Lukeino, 5,8 à 5,7 Ma). Kibingor (formation de Mabaget, 4,5 Ma). Koimogul (formation de Lukeino, 5,7 Ma). ? Mosionin (formation de Mabaget, 5 à 4,5 Ma). Sagatia (formation de Mabaget, 5 à 4,5 Ma). Tabarin (formation de Mabaget, $5 \mathrm{Ma}$ ).

Matériel: un ensemble constitué d'un crâne écrasé très fracturé, avec sa M2/ gauche détachée, et de sa mandibule, est conservé in situ à Kapsomin; 5 jugales supérieures isolées de Kapsomin n BAR 1904'01 (M2/ droite), 1906'01 (P4/ gauche), 1907'01 (P3/ droite), 1908'01 (P3/ gauche) et 1207'02 (P1/ gauche); une P2/ droite isolée n BAR 313'99 (Tabarin); fragment de molaire supérieure (27/9/99, Cheboit); fragment de jugale supérieure ${ }^{\circ}$ BAR 742'99 (Sagatia); ensemble $n^{\circ}$ BAR 1074'99 de fragments d'au moins 4 jugales inférieures (Tabarin); une M/2 gauche isolée $n^{\circ}$ BAR 226'99 (Kibingor); humérus incomplet $n^{\circ}$ BAR 549'03 (Kapchepkweren); fragment proximal de radius droit $n^{\circ}$ BAR 314'01 (Cheboit); magnum incomplet $n^{\circ}$ BAR 1719'01 (Kapchebrit); deux pyramidaux $\mathrm{n}^{\circ} \mathrm{BAR} 496^{\prime} 01$ et 273'00 (Cheboit); Mc III gauche (Fig. 6 A-C) n BAR 924'99 + 926'99 (Kapcharar); Mc IV gauche (Fig. 6 D-F) n BAR 1896'01 (Kapsomin); fragment distal de tibia droit $\mathrm{n}^{\circ}$ BAR 551'03 (Kapchepkweren); moitié médiale d'un astragale gauche ${ }^{\circ}$ BAR 1544'01 (Sagatia); Calcanéum gauche (Fig. 7 A-D) n BAR 90'98 (Kapchebrit); Calcanéum gauche n BAR 1899'01 (Kapsomin); Calcanéum gauche $\mathrm{n}^{\circ} \mathrm{BAR} 1125^{\prime} 02$ (Koimogul); extrémité distale de métapode axial n BAR 632’02 (Mosionin).

Description: Le crâne de Kapsomin est trop abîmé pour autoriser beaucoup d'observations; on voit néanmoins que les nasaux très épais, bombés, très arrondis, sont typiques de Ceratotherium. La longueur de l'échancrure nasale atteint $185 \mathrm{~mm}$, et la distance du fond de l'échancrure nasale à l'orbite $169 \mathrm{~mm}$. Ces deux variables valent respectivement $167 \mathrm{~mm}$ (un spécimen) et 172 à $185 \mathrm{~mm}$ (4 spécimens) chez les $C$. praecox pliocènes. L'avant de l'orbite se situe sur l'arrière de M1/, c'est une position avancée par rapport aux individus de Kanapoi et Ekora (avant de M2/) et surtout de Hadar et Laetoli (arrière de M2/). Rappelons que sa position normale chez l'actuel $C$. simum est entre le milieu de M2/ et l'avant de M3/ (Guérin, 1980).

Seule la branche horizontale droite de la mandibule de Kapsomin est étudiable; elle est plus haute que pour les $C$. praecox pliocènes: $126 \mathrm{~mm}$ entre $\mathrm{P} / 4-\mathrm{M} / 1$ (pour 6 spécimens, 92 à 115 $\mathrm{mm}$, moyenne $103,7 \mathrm{~mm}$ ), et $133,5 \mathrm{~mm}$ entre $\mathrm{M} / 2-\mathrm{M} / 3$ (pour 11 spécimens, 97 à $122 \mathrm{~mm}$, moyenne 110,3 mm). L'arrière de la symphyse est au niveau du milieu de $\mathrm{P} / 3$; pour 8 mandibules de l'Afar, cette position varie du milieu de $\mathrm{P} / 2$ à l'arrière de $\mathrm{P} / 3$. La symphyse haute de $62 \mathrm{~mm}$ a une section axiale en ellipse épaisse, presque circulaire.

Les M2/ de Kapsomin, d'usure moyenne, portent un cingulum labial discontinu; celle détachée du crâne conservé in situ possède un gros cingulum lingual, celle retrouvée isolée n'en a pas. L'ectolophe apparemment plat est en fait plus ou moins ondulé, avec un pli du paracône petit mais net et un fantôme de 

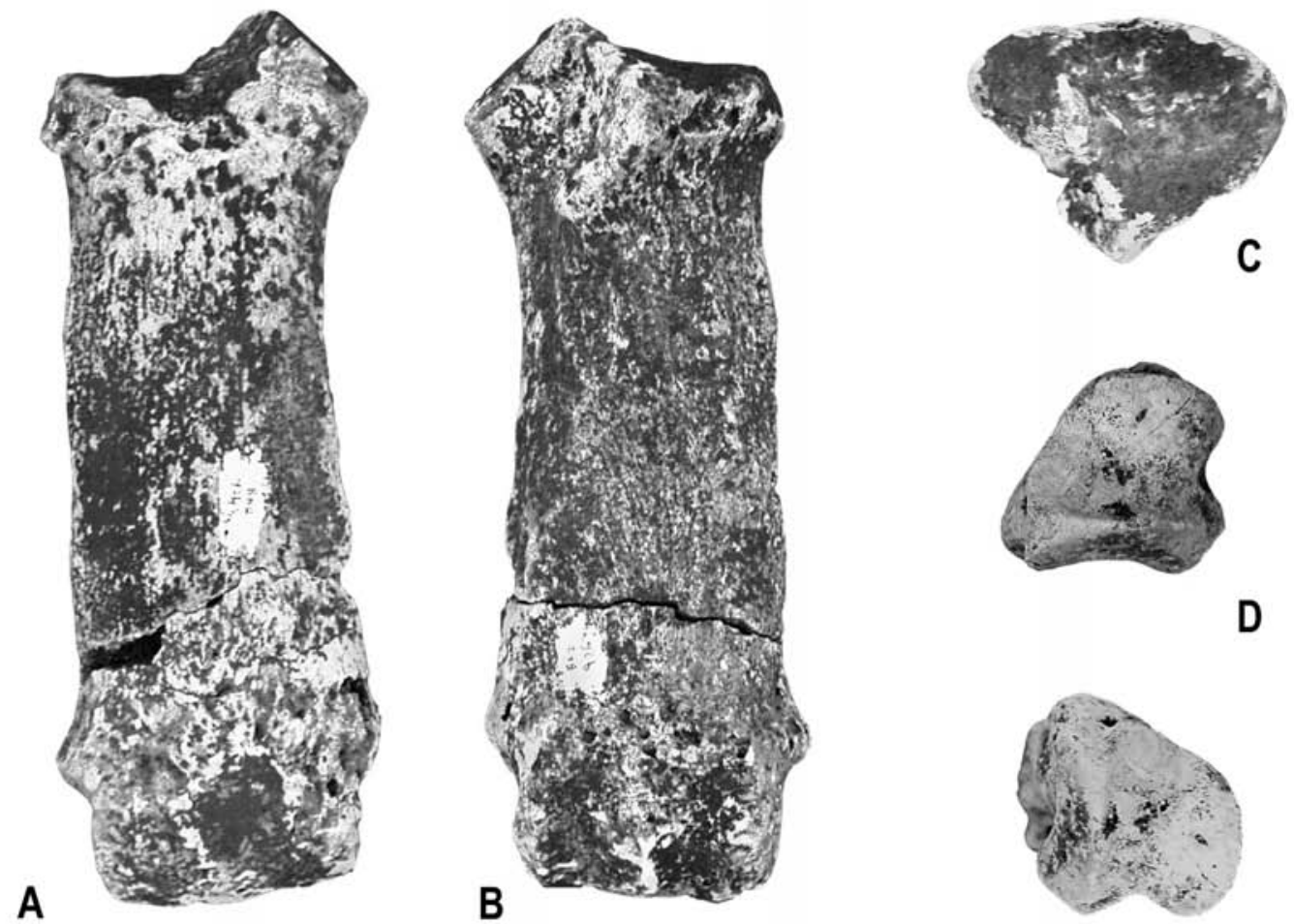

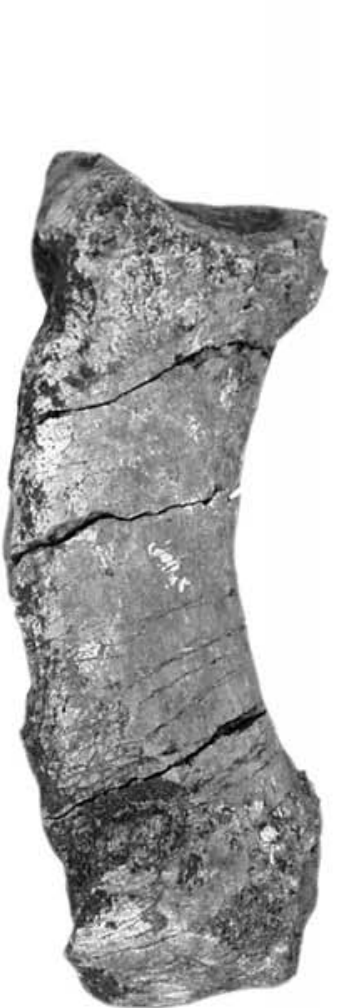

E

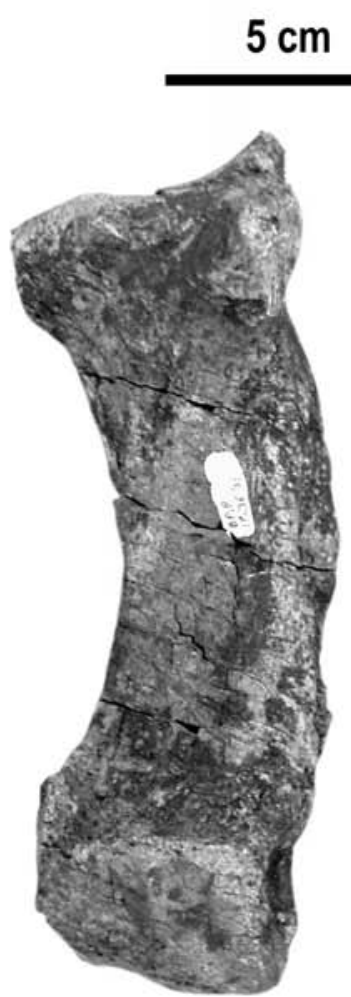

$\mathbf{F}$

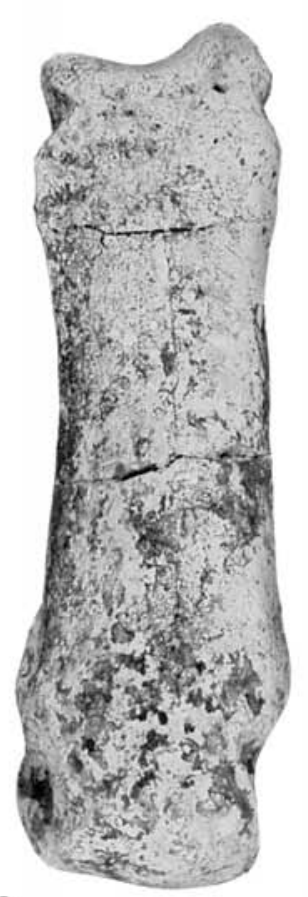

G

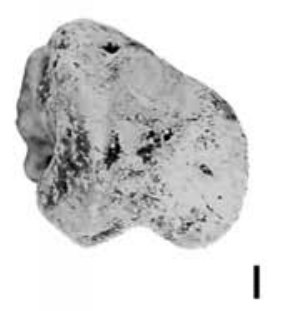

Fig. 6.-Ceratotherium praecox et Diceros cf. pachygnathus. A-C: Ceratotherium praecox, métacarpien III gauche de Kapcharar, $\mathrm{n}^{\circ}$ BAR 924'99 + 926'99; A: vue de face; B: vue postérieure. C: vue proximale. D-F: Ceratotherium praecox, métacarpien IV gauche de Kapsomin, n BAR 1896’01; D: vue proximale; E: vue de face; F: vue postérieure. G-I: Diceros cf. pachygnathus, métacarpien II gauche de Ngetabkwony, n BAR 298'00; G: vue antérieure; H: vue postérieure; I: vue proximale. 

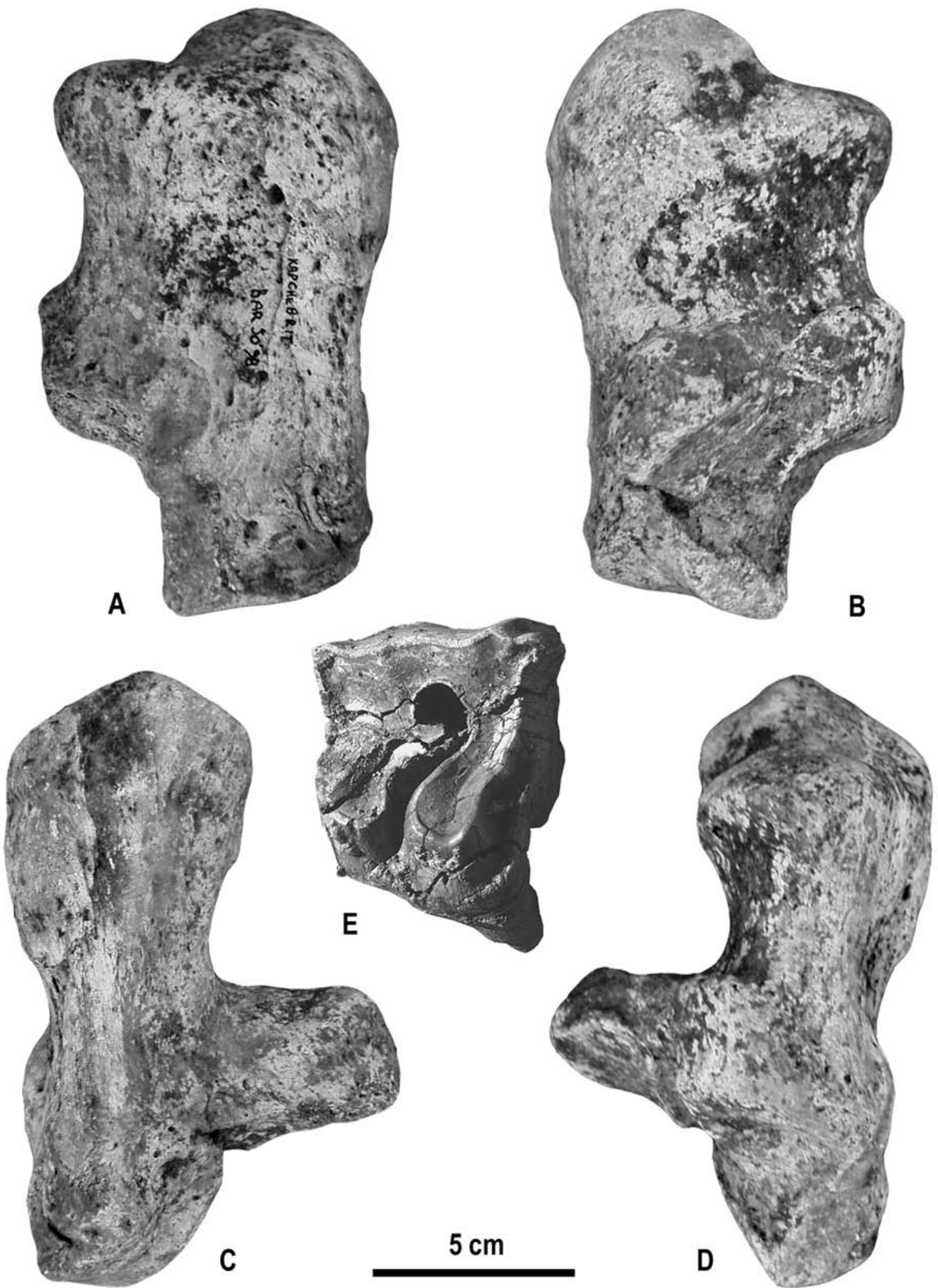

Fig. 7.-Ceratotherium praecox. A-D: Calcanéum gauche de Kapchebrit, n BAR 90'98; A: vue latérale; B: vue médiale; C: vue postérieure; D: vue de face. E: M 2/ droite de Kapsomin, sans $n^{\circ}$. Vue occlusale. 
Tableau 10.-Dimensions comparées du Mc Il des Rhinocerotidae néogènes des Tugen Hills

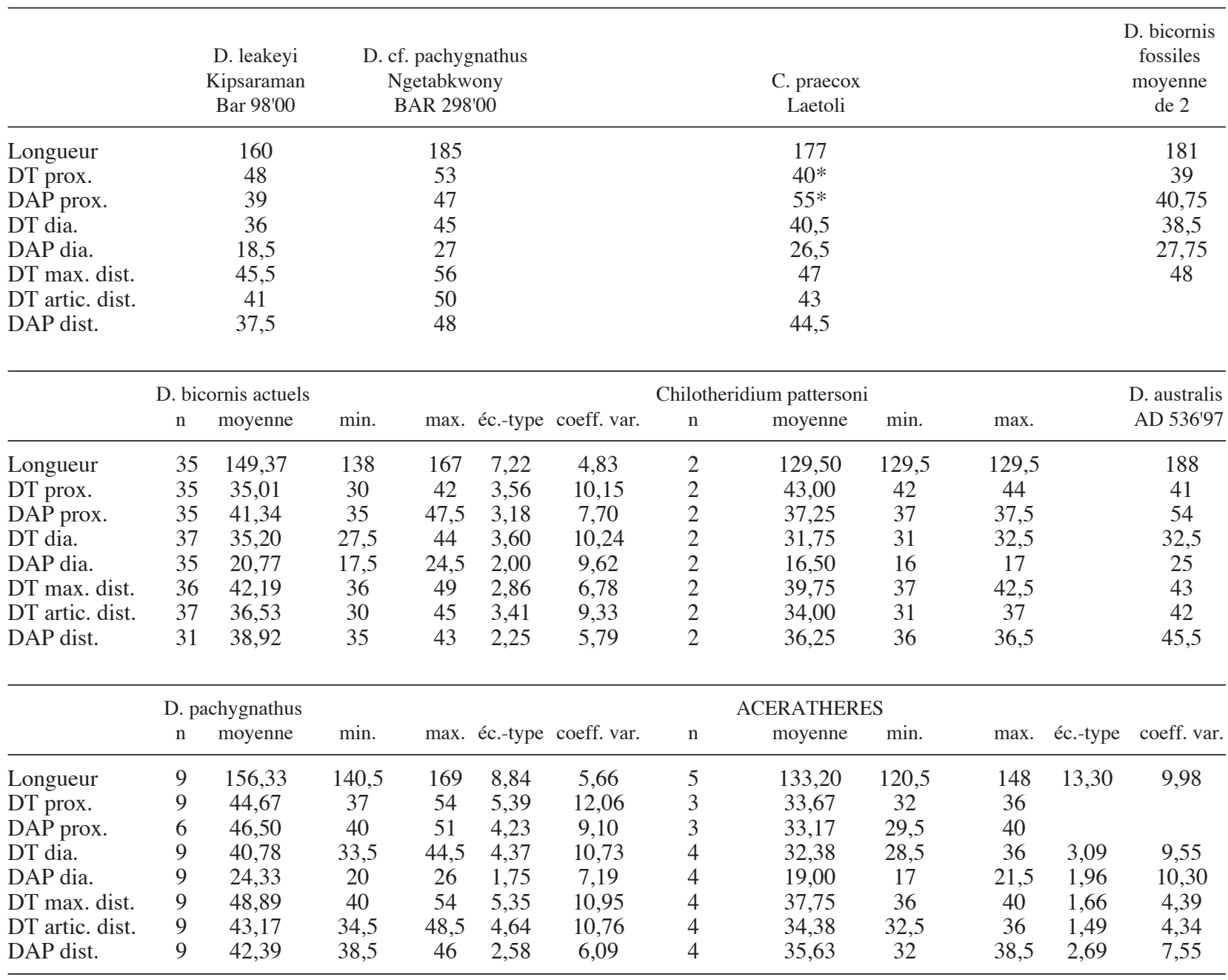

mésostyle. Le crochet est le seul repli interne. Le protocône est étranglé, plus faiblement sur la dent détachée du crâne (Fig. 7 E). Les dimensions sont données tabl. 4: elles correspondent parfaitement à celles des plus grands $C$. praecox d'Hadar et de Laetoli, chez qui la crista est souvent présente, la médifossette fréquemment fermée et le cingulum lingual parfois présent mais discontinu.

Le fragment de molaire supérieure de Cheboit est remarquable par sa grosse taille, son pli du paracône petit mais net apparaissant assez loin du collet, et son hypsodontie évidente en dépit de l'usure.

Le fragment de jugale supérieure de Sagatia n'est pas mesurable; je l'attribue à $C$. praecox du fait de son hypsodontie et de son ectolophe plat.

La P1/ de Kapsomin, très usée, est à section triangulaire; l'ectolophe est complexe, avec un très faible pli au tiers de sa longueur; aucune structure interne n'est observable. Les dimensions (tabl. 4) sont celles de $C$. praecox.

La P2/ de Tabarin est trop usée pour être étudiable, et sa longueur n'est pas significative. En revanche sa largeur, prise au collet et donc indépendante de l'usure, dépasse légèrement la valeur maximale observée sur un échantillon de 7 C. praecox de Hadar et de Laetoli (tabl. 4)

Les P3/ de Kapsomin sont usées. Elles sont munies d'un très fort cingulum lingual continu. L'ectolophe est faiblement et régulièrement convexe, sans pli du paracône visible. Le crochet est petit, il n'y a ni crista, ni anticrochet. Le protocône n'est pas étranglé.

La P4/ de Kapsomin, bien usée, porte un cingulum lingual continu. L'ectolophe est sub-plat. Le crochet est le seul repli interne. Il n'y a pas d'étranglement du protocône. La largeur (tabl. 4) est légèrement plus forte que la valeur maximale observée sur un échantillon d'une dizaine de $C$. praecox pliocènes, qui par ailleurs ont souvent une crista et une médifossette fermée, et chez qui le cingulum lingual est plus souvent absent que présent.

La rangée dentaire inférieure portée par la partie droite de la mandibule de Kapsomin montre des dimensions supérieures à celles des plus grands spécimens de $C$. praecox pliocènes (tabl. $5)$. $\mathrm{P} / 4$ et les deux premières molaires ont des vallées à profil transversal en V (la vallée postérieure de la M/3 est en U), avec une forte différence de niveau au dessus du collet entre vallée 
Tableau 11.-Dimensions comparées du Mc III des Rhinocerotidae néogènes des Tugen Hills

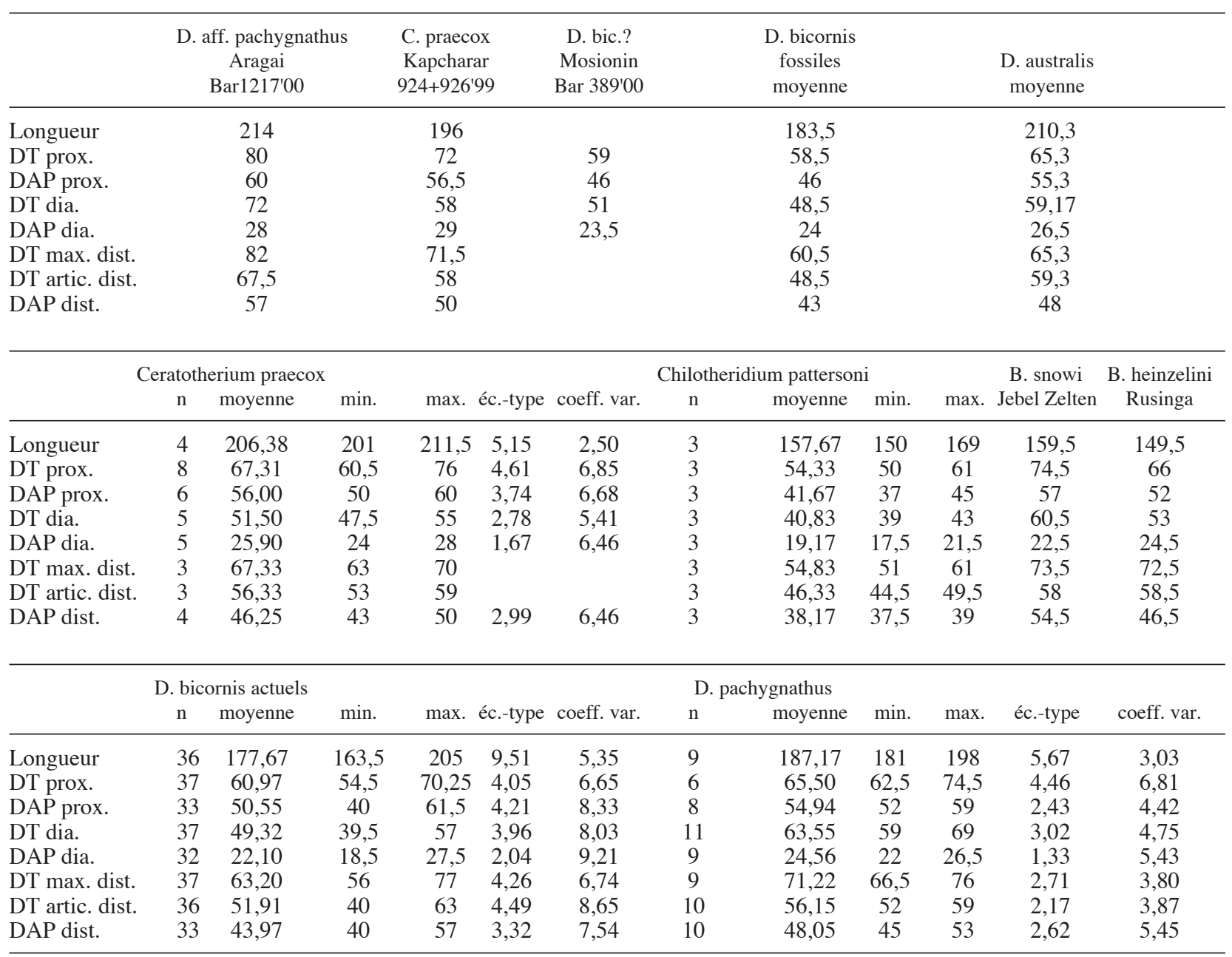

antérieure et vallée postérieure; les hauteurs des vallées sont semblables pour la P/4 et les molaires. Il n'existe pas de cingulum lingual.

La molaire inférieure de Kibingor, d'usure moyenne, est hypsodonte. Le synclinal labial est net. Les deux vallées linguales en $\mathrm{V}$ ont une faible différence de niveau. Il existe au niveau du collet un cingulum lingual et un cingulum labial. Les dimensions (tabl. 5), notamment la largeur, correspondent aux limites supérieures observées chez C. praecox.

Les fragments de jugales inférieures de Tabarin témoignent d'une grande taille, d'une forte hypsodontie et d'un synclinal labial bien marqué.

L'épiphyse proximale de radius de Cheboit, mal conservée, roulée et fissurée, a les dimensions et les proportions de $C$. praecox (tabl. 6).

Le magnum de Kapchebrit est incomplet en arrière. La hauteur sus-articulaire est $62 \mathrm{~mm}$, la hauteur de la face antérieure atteint $38 \mathrm{~mm}$, la largeur de cette face est égale ou supérieure à $43 \mathrm{~mm}$. Ces dimensions sont celles des C. praecox de Hadar et de Laetoli.

Les deux pyramidaux de Cheboit ont des dimensions (tabl. 8) correspondant bien à $C$. praecox.
Le Mc III de Kapcharar (Fig. 6 A-C) présente des dimensions (tabl. 11) et des proportions (Fig. 8) proches de celles des C. praecox pliocènes, et ne s'en distingue que par un diamètre transversal de la diaphyse un peu plus fort. La face proximale (Fig. 6 C) est large, avec un bord antérieur faiblement convexe mais légèrement déprimé au contact des facettes répondant respectivement au magnum et à l'onciforme. En face latérale de l'épiphyse proximale les deux facettes répondant au Mc IV sont bien distantes, la postérieure en triangle arrondi est haute et étroite. La facette supéro-médiale pour le Mc II est petite, allongée et sinueuse. La face antérieure (Fig. 6 A), sur laquelle la facette pour le magnum est bien visible sous cet angle, est large avec deux tubérosités à l'extrémité distale de la diaphyse, la tubérosité médiale étant plus saillante que la latérale; les deux tubérosités s'observent mieux en vue postérieure (Fig. 6 A B). La section de la diaphyse est trapézoïdale avec une face antérieure régulièrement bombée et une face postérieure régulièrement déprimée.

Le Mc IV de Kapsomin (Fig. 6 D-F) est a peine plus grand que le plus fort spécimen de 4 C. praecox pliocènes (tabl. 12) et ses proportions sont très voisines, sauf pour les diamètres antéropostérieurs des deux épiphyses, sensiblement plus élevés 


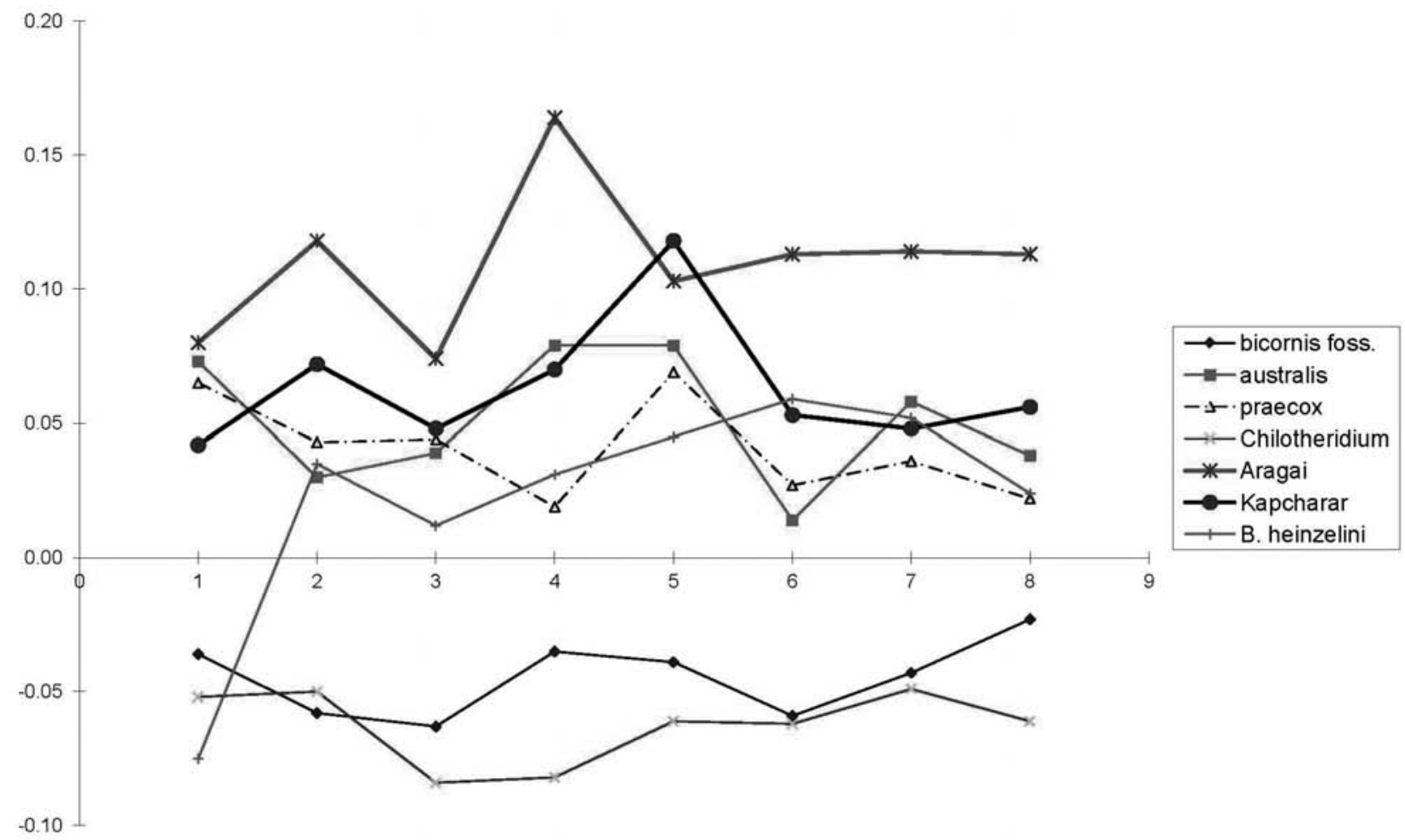

Fig. 8.-Diagramme des rapports des Mc III des Rhinocerotidae néogènes des Tugen Hills. Le référentiel est Diceros bicornis.Les variables en abscisse sont, dans l'ordre, celles du tabl. 11.

(Fig. 9 et 10). L'articulation proximale (Fig. 6 D) est triangulaire avec le sommet vers l'avant. L'articulation proximo-médiale possède deux facettes, l'antérieure basse et allongée, semielliptique, inclinée à $45^{\circ}$ vers l'avant et le bas, la postérieure bien distincte, en demi-cercle, portée par un pédoncule bien dégagé du corps de l'os. La vue antérieure (Fig. 6 E) met en évidence la largeur de la diaphyse et la courbure de ses extrémités vers l'extérieur, ce que montre aussi la face postérieure (Fig. 6 F); sous cet angle apparaît aussi une tubérosité médiale à l'extrémité distale de l'épiphyse.

Le fragment distal de tibia de Kapchepkweren est de très grande taille, notamment pour son diamètre transversal distal (tabl. 13). En face antérieure la coulisse des extenseurs des doigts est aussi large et peu profonde que chez les Dicerotinae actuels.

La moitié médiale d'astragale de Sagatia autorise la mesure de 4 variables, la hauteur médiale $(80,5 \mathrm{~mm})$, le diamètre antéropostérieur médial $(57,5 \mathrm{~mm})$, la hauteur de l'os au milieu de la poulie $(70 \mathrm{~mm})$ et le diamètre antéro-postérieur de l'articulation distale (environ $50 \mathrm{~mm}$. Ces valeurs se situent dans les limites d'un échantillon de 10 à 16 C. praecox de Hadar et de Laetoli.

Le calcanéum de Kapchebrit, bien conservé (Fig. 7 A-D), possède un contour latéral typique des Dicerotinae, globuleux, avec le bec constituant le point le plus antérieur, et un bord postérieur d'abord très convexe dans sa partie supérieure, puis faiblement déprimé à mi-hauteur (Fig. $7 \mathrm{~A}$ et $\mathrm{B}$ ). Il n'y a pas de facette répondant au péroné. La face articulaire postéro-inférieure est vaste, creusée transversalement et bombée longitudinalement. En vue caudale (Fig. 7 C) l'axe du sustentaculum est légèrement relevé vers le haut. Les dimensions (tabl. 14) sont celles d'un grand $C$. praecox. Le calcanéum de Koimogul est très semblable au précédent, dont il ne se distingue guère que par son très fort diamètre antéropostérieur. Le calcanéum de Kapsomin est incomplet (le sustentaculum tali est brisé), en mauvais état et recollé. Ses proportions sont celles de $C$. praecox mais ses dimensions sont nettement plus fortes que chez le plus grand spécimen pliocène de cette espèce (tabl. 14).

C'est surtout du fait de sa taille que j'attribue l'extrémité distale de métapode axial de Mosionin à C. praecox.

Affinités: $C$. praecox est donc pour l'instant connu en Afrique orientale, centrale et australe dans une période de temps comprise entre environ 7 et 3 à 2,5 millions d'années. C'est très probablement l'ancêtre des C. mauritanicum, $C$. germanoafricanum et C. simum du Quaternaire, qui ne feront qu'exprimer encore plus ses tendances évolutives. C'était un habitant de la savane, tondeur d'herbes, et tout particulièrement de graminées.

Il semble que les spécimens miocènes soient plus forts que ceux du Pliocène.

\section{Genre Diceros Gray, 1821}

Diceros aff. pachygnathus (Wagner, 1848; Gaudry, 1862-67)

Il s'agit d'une espèce originellement mal définie dont la synonymie est complexe. L'holotype est une mandibule juvénile découverte à Pikermi (MN 12, Grèce) et conservée à Munich. K. Heissig (1975) l'attribue à l'autre espèce de rhinocéros présente à Pikermi, Dicerorhinus orientalis (Schlosser, 1921), 


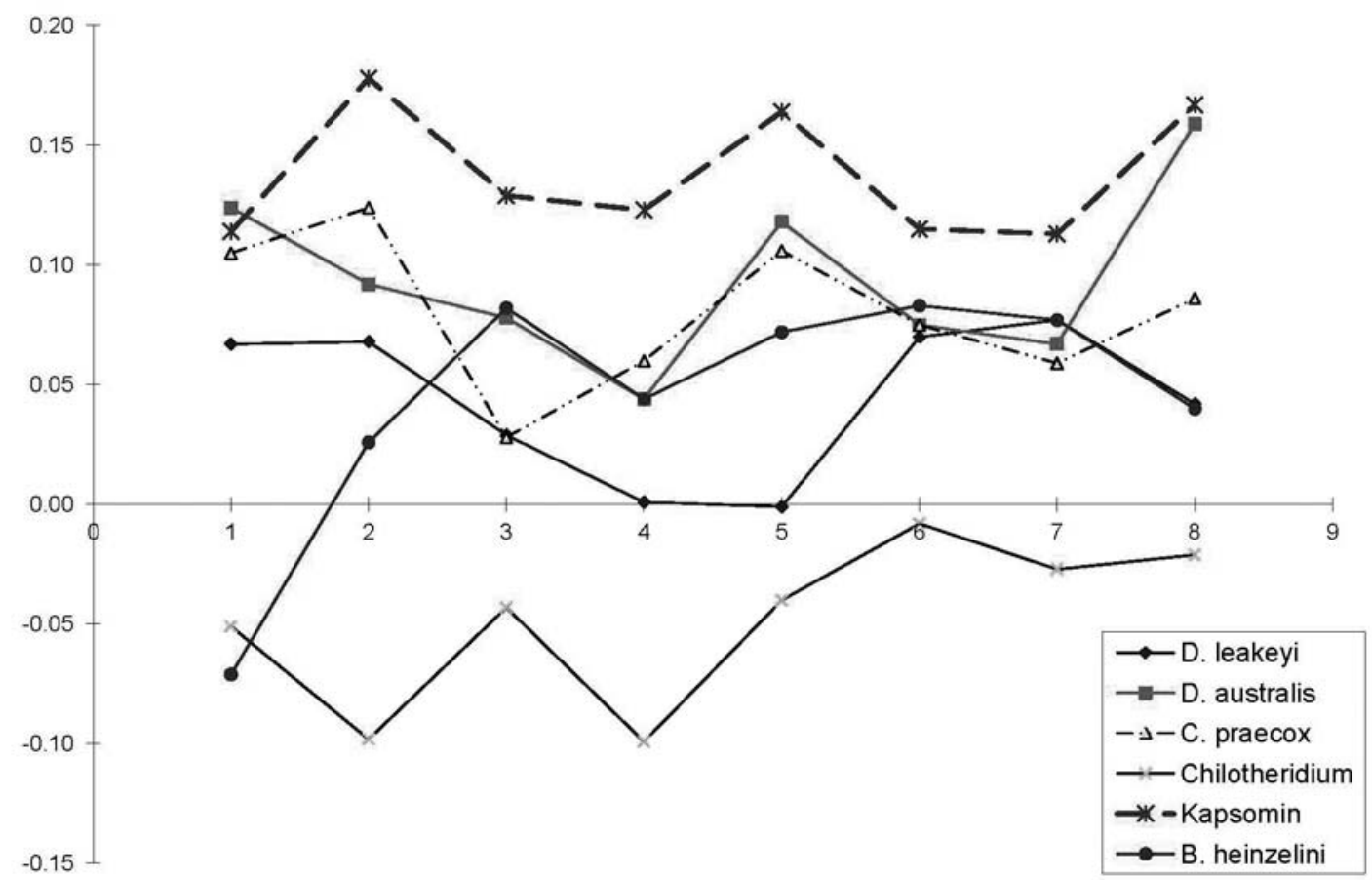

Fig. 9.-Diagramme des rapports des Mc IV des Rhinocerotidae néogènes des Tugen Hills (première partie). Le référentiel est Diceros bicornis. Les variables en abscisse sont, dans l'ordre, celles du tabl. 12.

\section{Mc IV bis}

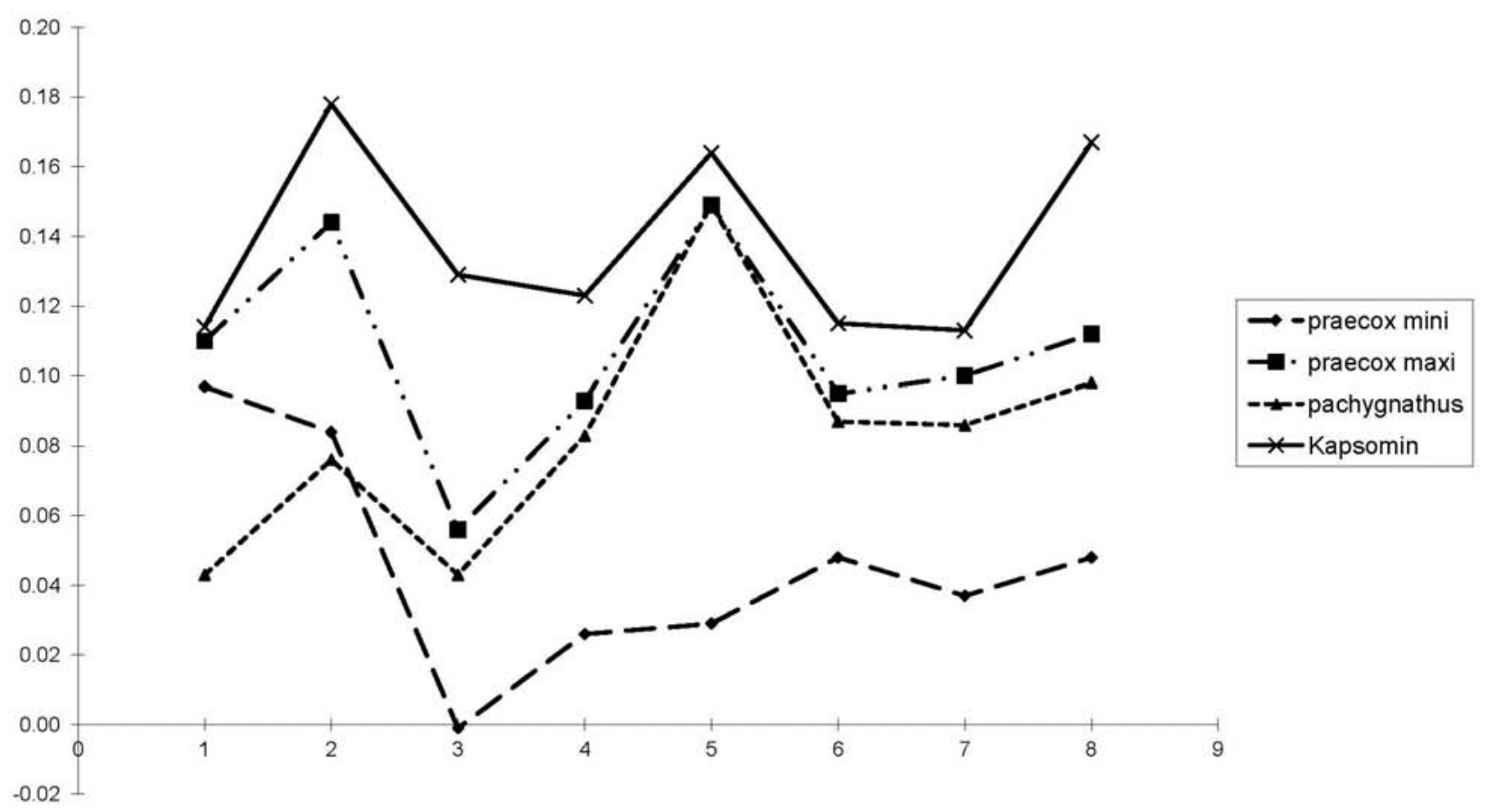

Fig. 10.-Diagramme des rapports des Mc IV des Rhinocerotidae néogènes des Tugen Hills (deuxième partie). Le référentiel est Diceros bicornis. Les variables en abscisse sont, dans l'ordre, celles du tabl. 12. 
Tableau 12.-Dimensions comparées du Mc IV des Rhinocerotidae néogènes des Tugen Hills

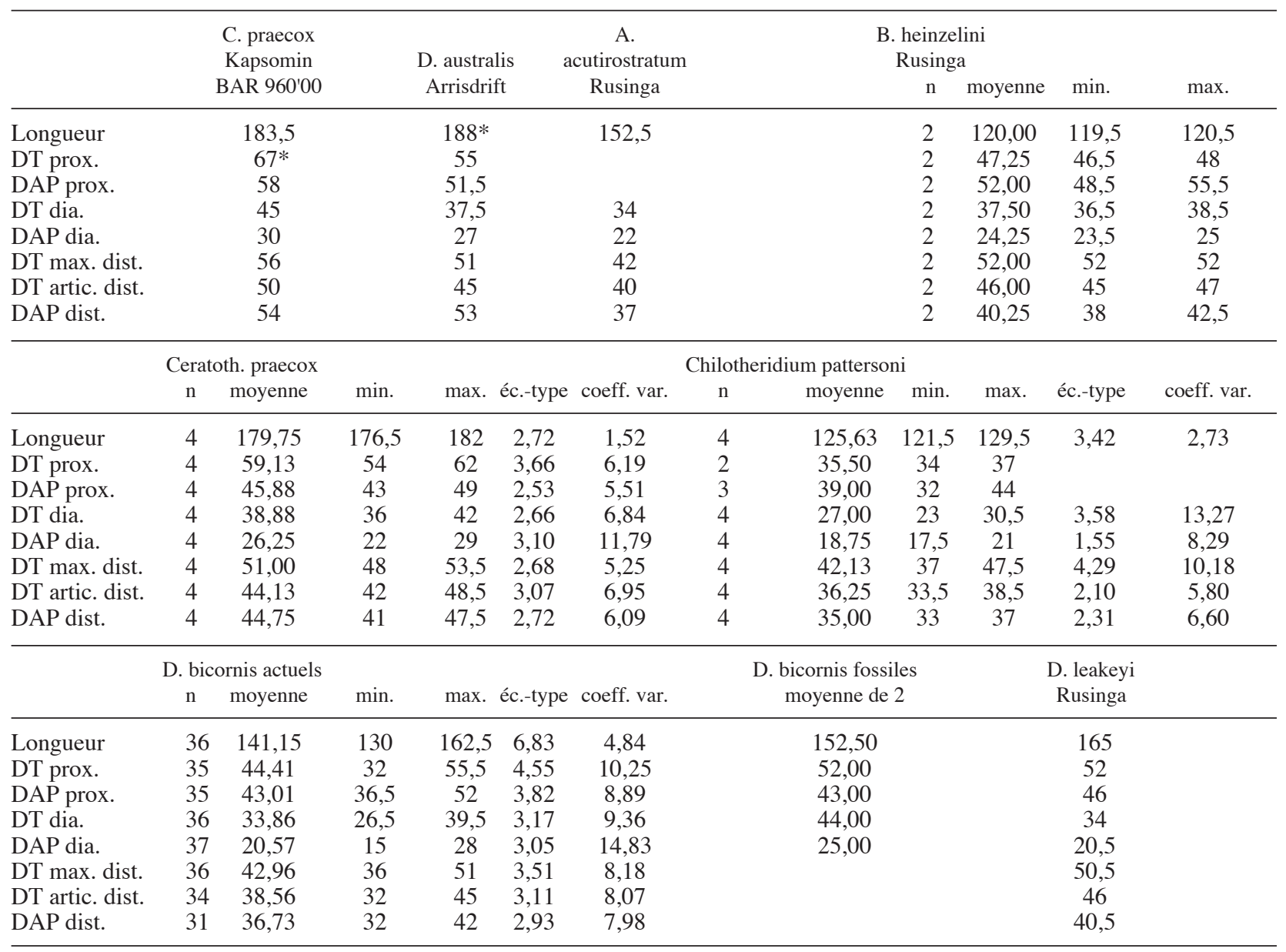

originellement déterminée comme «Rhinoceros» (ultérieurement Dicerorhinus) schleiermacheri, forme classique du Miocène supérieur d'Europe occidentale, puis à une sous-espèce particulière de celui-ci (D. s. orientalis), plus tard réévaluée au rang d'espèce. D. Geraads (1988) estime qu'il est impossible de rapporter l'holotype à l'une ou l'autre de ces espèces et propose d'en abandonner les noms au profit respectivement de Diceros neumayri (Osborn, 1900), suivant en cela K. Heissig (1975), et Dicerorhinus pikermiensis (Toula, 1906). Ce choix ne me paraît guère compatible avec le souci de stabilité de la nomenclature clairement exprimé par le Code international de nomenclature zoologique; de plus, et sans traiter ici de Dicerorhinus orientalis ni épiloguer sur la validité des nombreux noms d'espèces proposés par Toula, il ne me paraît pas nécessaire. En effet, Diceros pachygnathus a été longuement décrit par A. Gaudry (1862-67) sur du matériel cette fois indiscutable parmi lequel il suffit de désigner un lectotype. Le crâne avec sa mandibule figuré pl. XXVII de A. Gaudry fait parfaitement l'affaire et je propose donc sa désignation comme lectotype de Diceros pachygnathus.

Diceros neumayri (Osborn, 1900) a été défini à Maragha (Iran). S'il est identique à $D$. pachygnathus, comme le pense D. Geraads (1988), ce n'en est qu'un synonyme récent. Je suis quant à moi d'accord avec E. Thenius (1955) qui en a fait une sous-espèce de $D$. pachygnathus. $D$. pachygnathus pachygnathus et $D$. pachygnathus neumayri ont un squelette post-crânien très semblable et se distinguent surtout par le plus ou moins net applatissement de l'ectolophe des jugales supérieures au niveau du pli du paracône: $D$. p. neumayri possède un ectolophe plus plat et est plus hypsodonte. K. Heissig (1975) reconnaît d'ailleurs à partir de ces critères l'existence de deux variantes de D. neumayri mais ne les distingue pas sur le plan systématique.

Il existe donc au Miocène supérieur un groupe d'espèces périméditerranéennes de Diceros, comprenant $D$. pachygnathus du Proche- et du Moyen-Orient (Grèce, Turquie, Iran) avec deux sous-espèces, et dont font aussi partie $D$. douariensis Guérin, 1966, de Tunisie et d'Italie (Baccinello V 3, Guérin 2000), et les D. cf. pachygnathus d'Autriche (bassin de Vienne, Thenius, 1956), d'Espagne (Cenes de la Vega et Los Hornillos, tous deux du bassin de Grenade, Guérin, 1980 a) et d'Italie (Gravitelli en Sicile, Hooijer, 1946). En 1982 K. Heissig attribue à «cf. $D$. neumayri» une deuxième prémolaire supérieure et peut-être une deuxième phalange axiale découvertes à Sahabi (Libye); il n'envisage pas que ce matériel puisse être attribué à D. douariensis, pourtant géographiquement plus proche. Cette détermination est reprise en 1987 par R.L. Bernor et al., qui 
Tableau 13.-Dimensions comparées du tibia des Rhinocerotidae néogènes des Tugen Hills

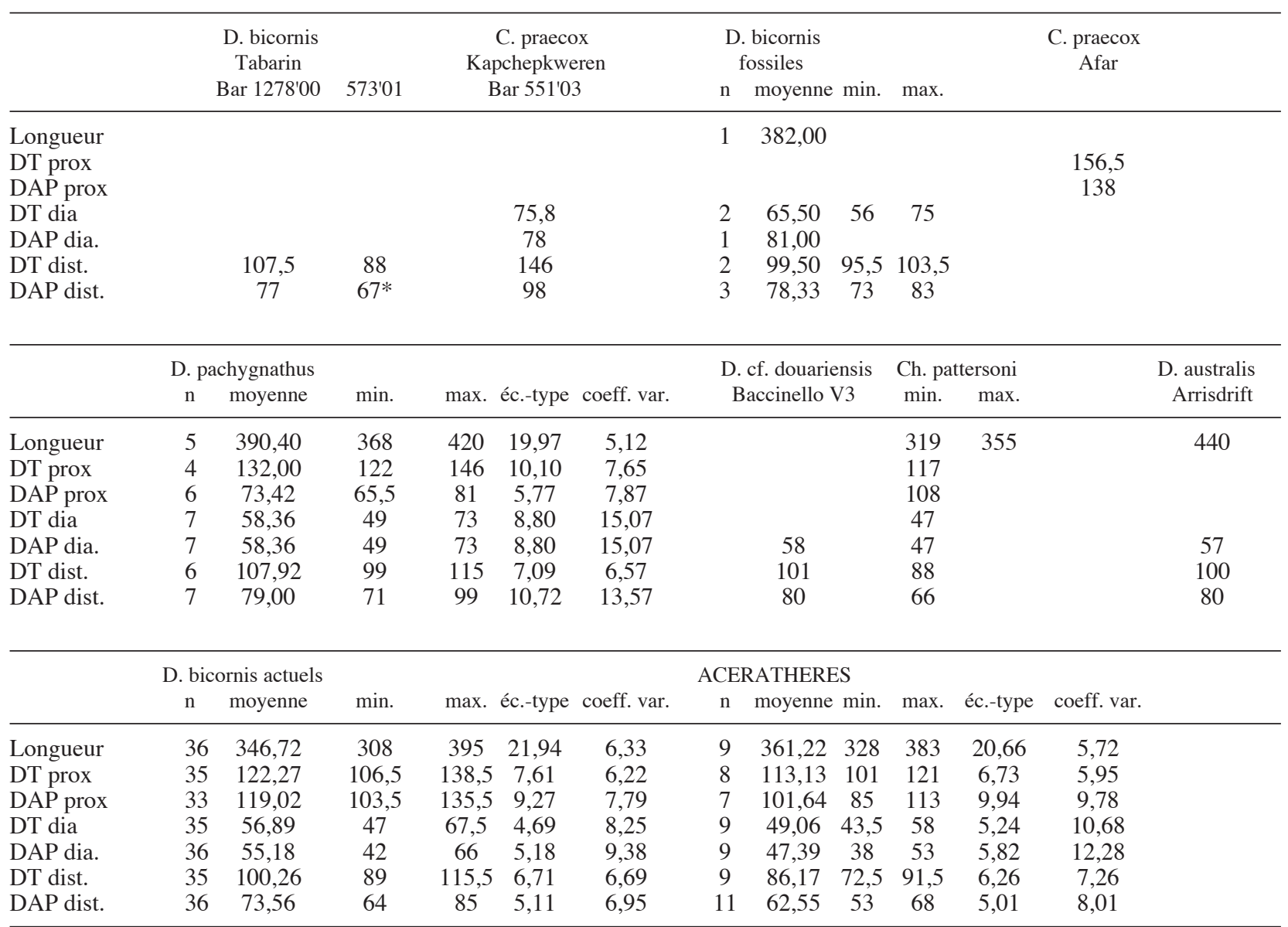

figurent la prémolaire et attribuent à tort la paternité du taxon neumayri à de Mecquenem, 1905.

Il existe aussi un problème d'attribution générique. D. Geraads (1988) considère que «l'ancêtre commun aux deux espèces africaines actuelles, dont $C$. neumayri n'est sans doute pas très éloigné, ressemblait beaucoup plus à Ceratotherium qu'à Diceros, genre qui pourrait être bien plus récent». Il attribue donc ces espèces miocènes au genre Ceratotherium, en se fondant sur trois caractères, la longueur de l'apophyse posttympanique, la forme des nasaux et la position reculée du fond de l'échancrure nasale. Il ne tient en revanche aucun compte de bien d'autres éléments pourtant caractéristiques du genre, en particulier de la morphologie dentaire et de l'hypsodontie. Il semble que cette interprétation, reprise en 1990 par D. Geraads \& G. Koufos, inverse la polarité des caractères et prenne à rebours le sens de l'évolution chez les Dicerotinae. Le genre Diceros, connu maintenant en Afrique depuis la base du Miocène moyen (Guérin, 2000, 2003), est en fait beaucoup plus ancien que Ceratotherium apparu près de 12 millions d'années plus tard au Miocène supérieur, et il est comme il se doit beaucoup moins spécialisé. Par ailleurs Diceros bicornis, brachyodonte et ubiquiste, est désormais attesté dans le Miocène supérieur des Tugen Hills (voir ci-après); cette espèce est ainsi apparue sensiblement en même temps que le premier Cerato- therium, C. praecox, déjà franchement hypsodonte et adapté à la savane. Rien ne justifie l'attribution de Diceros pachygnathus au genre Ceratotherium.

Néodiagnose (Guérin, 1980 a, simplifiée): Diceros de taille grande à très grande. Crâne très grand à massif facial relativement court, nasaux courts, convexes et épais. Face occipitale oblique vers l'arrière et le bas, grande crête occipitale déprimée dans sa partie centrale; toît crânien à profil subrectiligne; pseudo-méat auditif fermé vers le bas.

Mandibule à symphyse brève; branche horizontale large, basse, à bord ventral légèrement convexe; branche montante à bord antérieur légèrement oblique vers l'avant.

Pas de denture antérieure fonctionnelle. Jugales brachyodontes; molaires supérieures à ectolophe presque plat, sans pli du paracône net; crochet présent, crista peu développée quand elle existe, anticrochet rare; étranglement du protocône toujours présent aux M1/ et 2/; cingulum lingual faible ou absent. P3/ et 4/ à ectolophe presque plat, avec plis du paracône et du métacône à peine sensibles; crochet toujours présent, crista fréquente, pas d'anticrochet, pas de médifossette fermée observée; pas d'étranglement du protocône; cingulum lingual normalement présent.

Squelette des membres typique du genre avec humérus allongé, radius plutôt court, métapodes longs et épais. 
Tableau 14.-Dimensions comparées du calcanéum des Rhinocerotidae néogènes des Tugen Hills

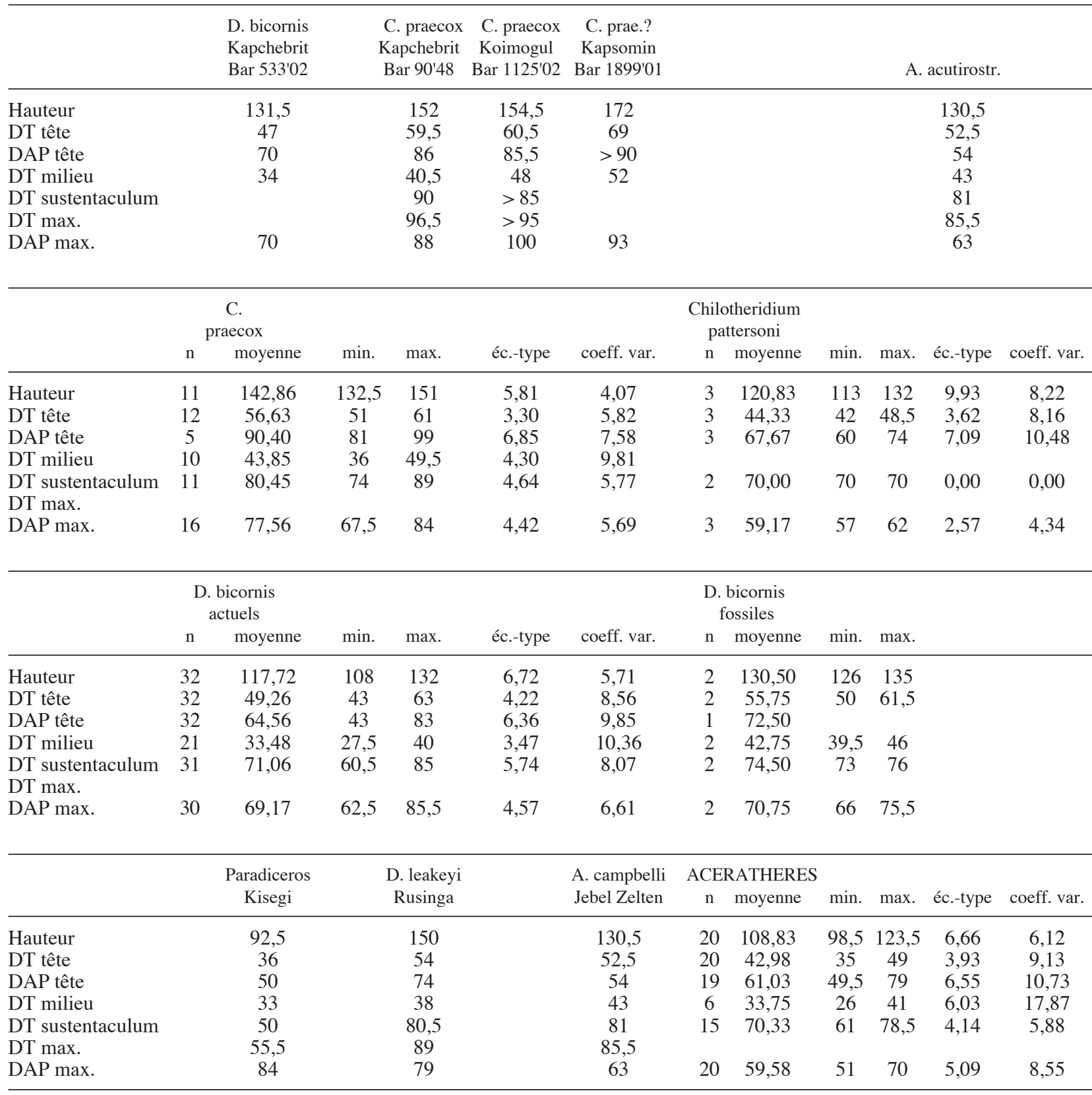

Gisements des Tugen Hills: Aragai (formation de Lukeino, 6 Ma). Ngetabkwony (formation de Mabaget, 5 à 4,5 Ma).

Matériel: M3/ gauche ${ }^{\circ}$ BAR 1587'00 (Aragai); Mc II gauche (Pl. 4 G-I) n BAR 298'00 (Ngetabkwony); Mc III gauche ${ }^{\circ}$ BAR $1217^{\prime} 00$ (Aragai).

Description: La M3/ d'Aragai, très usée, se caractérise par son émail très épais et son faible pli du paracône; elle ne possède pas de pli interne ni de cingulum lingual ou labial. Ses dimensions (tabl. 4) et sa morphologie correspondent à $D$. pachygnathus.
Le Mc II de Ngetabkwony (Fig. 6 G-I) est un peu plus grand et plus fort qu'un spécimen de C. praecox de Laetoli (tabl. 10). Ses proportions sont très semblables à celles de $D$. pachygnathus et bien différentes de C. praecox (Fig. 2 et 3), encore que l'unique spécimen de comparaison de cette dernière espèce soit mal conservé dans sa partie proximale et donc pas très significatif. En vue supérieure (Fig. 6 I) le bord le plus antérieur de l'articulation est très en retrait de l'avant de l'épiphyse proximale. L'articulation proximo-latérale est constituée d'une seule facette en croissant surmontant une profonde dépression; la facette est plane et ne présente aucun dièdre entre sa partie 
répondant au magnum et celle qui correspond au Mc III. Il n'y a pas d'articulation proximo-médiale. L'épiphyse proximale en vue de face (Fig. $6 \mathrm{G}$ ) porte deux nettes tubérosités, une latérale et une médiale. L'épiphyse distale en vue postérieure (Fig. $6 \mathrm{H}$ ) porte elle aussi deux tubérosités, et sa partie centrale est déprimée. La section de la diaphyse est en triangle arrondi avec une face antérieure convexe, un bord médial tranchant, une face postérieure subplane et une face latérale concavo-convexe.

Le Mc III d'Aragai, un peu roulé, est bien restauré (Fig. 11). L'articulation proximale (Fig. $11 \mathrm{C}$ ) se caractérise par son bord antérieur nettement déprimé, et sa limite antérieure en retrait du bord de l'os. En vue antérieure (Fig. 11 A) et postérieure (Fig. 11 B) l'os apparaît remarquablement large, en particulier dans sa partie inférieure; à son extrémité distale la diaphyse présente sur chacun de ses bords une nette tubérosité. La section de la diaphyse, large et plate, est trapézoïdale avec un bord antérieur convexe, un bord postérieur concave, un bord latéral rectiligne, un bord médial arrondi. Les dimensions (tabl. 11) dépassent les valeurs maximales observées pour un échantillon de 3 à $8 C$. praecox, notamment pour le diamètre transversal au milieu de la diaphyse et les diamètres transversaux sus-articulaire et articulaire de l'épiphyse distale, et les proportions sont différentes; ces proportions (Fig. 8) sont beaucoup plus proches des valeurs maximales observées sur un échantillon de 8 à $11 \mathrm{D}$. pachygnathus, que les dimensions du spécimen d'Aragai dépassent légèrement.

Affinités: La découverte de Diceros cf. pachygnathus est l'un des résultats les plus intéressants de l'étude des Rhinocéros des Tugen Hills. Elle étend largement vers le Sud l'extension géographique de cette espèce, et montre qu'elle est en Afrique sympatrique et à peu près contemporaine des premiers Diceros bicornis et Ceratotherium praecox, rendant très peu plausible un quelconque rapport de filiation entre ces trois rhinocéros.

\section{Diceros bicornis (Linné, 1758)}

Il s'agit de l'actuel rhinocéros «noir», espèce non spécialisée apparue au Miocène supérieur et qui n'a pas suffisamment évolué depuis pour justifier une distinction systématique de ses représentants néogènes.

Néodiagnose (Guérin, 1980 a, simplifiée): crâne à massif facial bref et intermaxillaires réduits, aux nasaux courts, épais et fortement convexes; pas de processus post-orbitaires; angle entre face supérieure et face occipitale faiblement aigu; pseudoméat auditif ouvert vers le bas. Mandibule à symphyse courte; branche horizontale à bord inférieur très courbe; branche montante à bord antérieur oblique vers l'arrière et le haut.

Pas de denture antérieure fonctionnelle. M1/ et M2/ avec pli du paracône moyen et faible dépression de l'ectolophe au niveau du métacône; crista en général absente, crochet presque toujours présent, médifossette exceptionnellement fermée; cingulum lingual discontinu, pouvant manquer aux M2/; étranglement du protocône assez fréquent. $\mathrm{P} 3$ / et $4 /$ avec pli du paracône plus ou moins saillant mais toujours très net; en arrière de ce pli le profil de l'ectolophe est relativement plat; crista présente ou absente, plus souvent présente aux $\mathrm{P} 4 /$; crochet presque toujours présent, souvent double ou bifide; médifossette fermée fréquente, surtout aux P4/; cingulum lingual bien marqué; étranglement du protocône exeptionnel aux $\mathrm{P} 3$ / et assez rare aux $\mathrm{P} 4 /$.

Jugales inférieures avec vallées linguales en $\mathrm{V}$ présentant une nette différence de niveau entre l'antérieure et la postérieu-
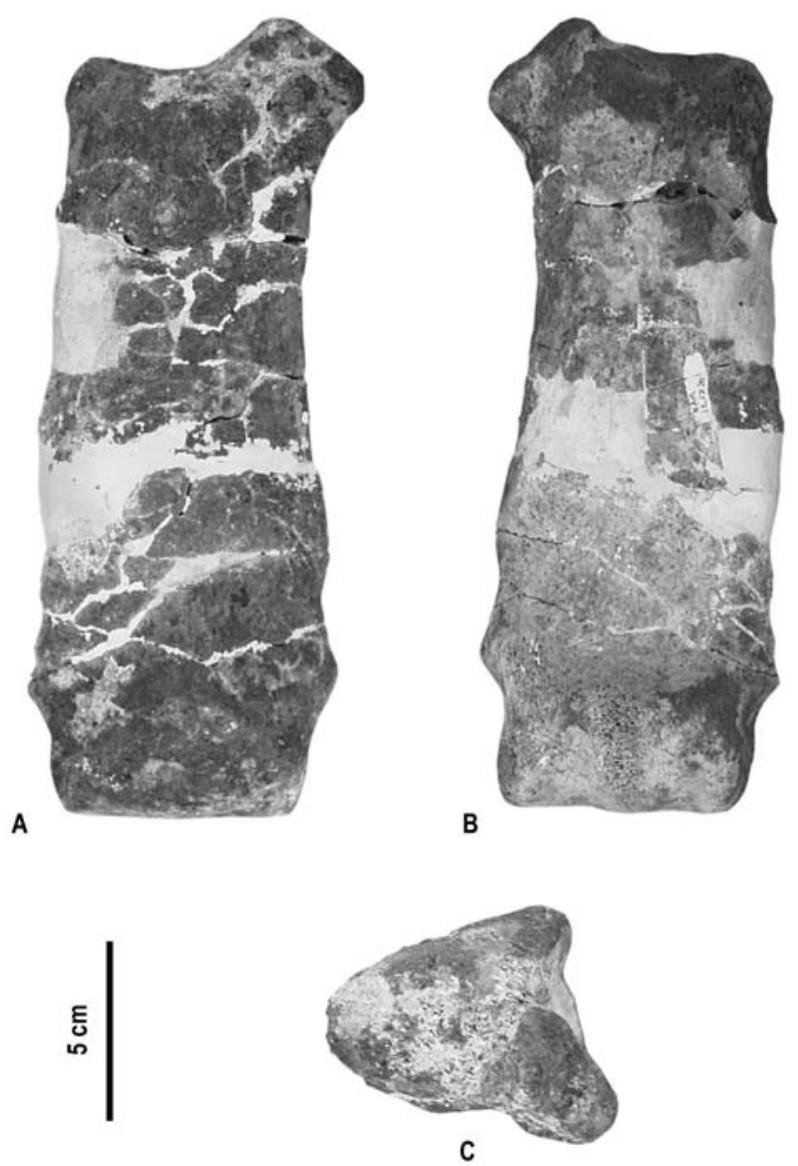

Fig. 11.-Diceros cf. pachygnathus de Aragai, métacarpien III gauche $n^{\circ}$ BAR 1217'00. A: vue antérieure. B: vue postérieure. C: vue proximale.

re, surtout pour les prémolaires; synclinal labial large et peu profond.

Squelette des membres caractéristique.

Gisements: D. bicornis est attesté en tant que fossile dans de nombreux gisements africains datant des 5 derniers millions d'années, notamment:

- Afrique du Sud: Elandsfontein, Hopefield (0,6 à 0,5 Ma); Langebaanweg 1 et 2 (5 Ma); Limeworks Cave, Makapansgat (3,4 à $3 \mathrm{Ma})$.

- Ethiopie: Hadar (3,5 à 2,8 Ma); formation de Konso (niveau 4) dans le Sud Ethiopien (1,4 Ma); Omo, dans la formation de Mursi (un peu plus de $4 \mathrm{Ma}$ ) et dans tous les membres (de A à K/L: 3,8 à 1,3 Ma) de la formation de Shungura.

- Kénya: Kanam (5 Ma); Kapthurin formation (0,7 à 0,4 Ma); Koobi Fora, formation de Kubi Algi (4 à 2,5 Ma); Kanjera (2,3 à 0,6 Ma); Malewa River (Pléistocène supérieur); Naivasha (Pléistocène supérieur).

- Mali: Région d'Araouane, site AR 7 (7000 ans BP) dans le Bassin de Taoudenni; c'est le site marquant la limite Nord-Occidentale d'extension de l'espèce (Guérin \& Faure, 1983). 
- Ouganda: Walasi Hill (Pléistocène) et formations de Behanga 1 (1,8 Ma), Nkondo (5 Ma), Nyabusosi (1,5 Ma) et Warwire (4,5 Ma).

- Tanzanie: Laetoli (3,8 à 3,5 Ma); Olduvai Beds I à IV (2,1 à $0,7 \mathrm{Ma})$

- Tchad: A. Likius (2002) signale l'espèce dans le Pliocène du Tchad à Kossom Bougoudi (autour de $5 \mathrm{Ma}$ ), à Kollé (5 à 4 Ma) et à Koro Toro (3,5 à $3 \mathrm{Ma})$.

- Zambie: Kabwe (= Broken Hill, 0,1 Ma).

Gisements des Tugen Hills: Kapchebrit, Mosionin, Sagatia, Tabarin (tous de la formation de Mabaget, 5 à 4,5 Ma). Mutuko (formation de Lukeino, $6 \mathrm{Ma}$ ).

Matériel: $\mathrm{M} / 2$ droite isolée ${ }^{\circ} \mathrm{BAR} 76^{\prime} 00$ (Mutuko); $\mathrm{P} / 2$ droite isolée $n^{\circ}$ BAR 1304'00 (Tabarin); scaphoïde droit $n^{\circ}$ BAR 1532'02 (Sagatia); tiers proximal d'un Mc III droit $n^{\circ}$ BAR 389'00 (Mosionin); deux tiers distaux d'un fémur gauche $\mathrm{n}^{\circ}$ BAR 682'02 (Tabarin); deux fragments distaux de tibias gauches $n^{\circ}$ BAR $1278^{\prime} 00$ et BAR 573'01 (Tabarin); calcanéum droit n ${ }^{\circ}$ BAR 533'02 (Kapchebrit).

Description: La molaire inférieure de Mutuko est d'usure moyenne; il n'y a pas de cingulum lingual ni labial. Les vallées linguales en $\mathrm{V}$ présentent une forte différence de niveau. En vue occlusale les croissants sont arrondis. Les dimensions (tabl. 5) correspondent parfaitement aux D. bicornis actuels.

La prémolaire inférieure de Tabarin est trop usée pour autoriser une description morphologique. Sa largeur, seule dimension exploitable, est proche de la moyenne d'un échantillon de $38 \mathrm{D}$. bicornis actuels.

Le scaphoïde de Sagatia a le contour globuleux, le bord antérieur déprimé et le bord postérieur très convexe caractéristiques du genre. Les dimensions (tabl. 7) sont presqu'identiques à la moyenne d'un échantillon de 6 à $7 \mathrm{D}$. bicornis plio-pléistocènes et se placent dans l'intervalle de variation d'un lot de 26 à 27 D. bicornis actuels. La hauteur de l'os en avant est à peu près identique à sa hauteur postérieure.

Le fragment proximal de Mc III de Mosionin présente les caractéristiques morphologiques, les dimensions et les proportions des D. bicornis actuels et fossiles (tabl. 11).

Le fémur incomplet de Tabarin a des dimensions et proportions (diamètre transversal minimal de la diaphyse $80,5 \mathrm{~mm}$; diamètre antéropostérieur de la diaphyse $69 \mathrm{~mm}$; diamètre transversal distal $145 \mathrm{~mm}$; diamètre antéropostérieur distal supérieur ou égal à $180 \mathrm{~mm}$ ) correspondant aux valeurs maximales observées sur un échantillon de $34 \mathrm{D}$. bicornis actuels.

Le plus gros (BAR 1278'00) des deux fragments distaux de tibias de Tabarin a des dimensions un peu supérieures à la moyenne d'un échantillon de 35 à 36 D. bicornis actuels; le plus petit (BAR 573'01) est proche des valeurs minimales de cet échantillon (tabl. 13).

Le calcanéum de Kapchebrit, dont le sustentaculum est brisé, montre le profil globuleux caractéristique de la sous-famille, moins marqué toutefois sur le bord postérieur que pour les $D$. bicornis actuels; l'avant de la tubérosité est moins développé antérieurement que le bec. Les dimensions (tabl. 14) se situent toutes dans l'intervalle de variation de D. bicornis.

Affinités: $D$. bicornis est désormais connu depuis 6 Ma, l'espèce est donc apparue à peu près en même temps que Ceratotherium praecox et a été en partie contemporaine des Diceros du groupe $D$. pachygnathus.

C'est un rhinocéros peu évolué, aux jugales relativement brachyodontes et au port de tête haut; sa lèvre supérieure en $\mathrm{V}$ est préhensile et lui sert à saisir les rameaux dont il se nourrit: épineux et buissons surtout, aussi diverses herbacées et fruits. Plutôt ubiquiste, il habite préférentiellement les régions sèches à buissons et la brousse à épineux.

\section{Conclusion}

Neuf espèces de rhinocéros sont attestées dans 26 gisements du Néogène des Tugen Hills. Aceratherium acutirostratum y est présent dans 4 sites, son squelette post-crânien est désormais identifiable et peut être distingué de celui de Dicerorhinus leakeyi qui existe en même temps que lui à Kipsaraman. Brachypotherium heinzelini se trouve dans le gisement de Cheparawa en même temps que A. acutirostratum et $P$. mukirii, et Brachypotherium lewisi est présent dans trois sites, où il est sympatrique de Ceratotherium praecox (et aussi de D. bicornis à Sagatia). Chilotheridium pattersoni est identifié dans un site de 9,5 Ma, ce qui étend de 2,5 Ma la durée de vie de l'espèce. Dicerorhinus leakeyi est reconnu dans 3 gisements, son squelette post-crânien est maintenant reconnaissable. Paradiceros mukirii représenté à Cheparawa par deux crânes permettant d'affiner la diagnose de l'espèce est donc désormais attesté à partir de 15,5 Ma. Ceratotherium praecox, présent dans au moins 12 sites depuis $6 \mathrm{Ma}$, est le rhinocéros le plus fréquent. Diceros cf. pachygnathus est reconnu à Aragai et Ngetabkwony; sa présence dans les formations de Lukeino et Mabaget, vers 6 et $5 \mathrm{Ma}$, accroît considérablement l'extension géographique, jusqu'ici périméditerranéenne, des espèces de ce groupe. Enfin Diceros bicornis est attesté dans 5 gisements dont le plus ancien, Mutuko, date de $6 \mathrm{Ma}$; il est sympatrique de C. praecox dans trois ou quatre de ces sites et semble apparaître à peu près en même temps que ce dernier. L'Hominidé Orrorin tugenensis est présent dans quatre sites de la formation de Lukeino datant de 6 à $5,7 \mathrm{Ma}$ (Senut et al., 2001); à Aragai il est associé à Diceros cf. pachygnathus; à Cheboit avec Ceratotherium praecox et Brachypotherium lewisi, à Kapcheberek à $C$. praecox; à Tabarin avec $C$. praecox et $D$. bicornis (tabl. 1). Ces associations montrent que l'environnement d'Orrorin était varié mais à dominante ouverte et plutôt humide.

\section{REMERCIEMENTS}

Brigitte Senut et Martin Pickford m'ont confié l'étude des Rhinocerotidae des Tugen Hills, m'ont emmené en 2002 sur le 
terrain et invité la même année au colloque international «From Samburupithecus to Orrorin: Origins Of Hominids. Geological and Palaeontological Background» qui s'est tenu à Bogoria (Kénya), où j'ai présenté une première ébauche du présent article. Madame Mary Muungu, responsable des collections, et Madame le Dr. Emma Mbua, directrice du Département de Paléontologie du National Museums of Kenya à Nairobi, m'ont permis d'accéder aux collections qu'elles dirigent. Martin Pickford a réalisé les photographies illustrant cet article, et Noël Podevigne a préparé et monté les planches. Jorge Morales a traduit le résumé en espagnol.

\section{Références}

Arambourg, C. (1959). Vertébrés continentaux du Miocène supérieur de l'Afrique du Nord. Publications Service Carte géologique de l'Algérie, ns, Paléontologie, mém. 4, p. 1-161.

Bernor, R.L.; Heissig, K. \& Tobien, H. (1987). 17. Early Pliocene Perissodactyla from Sahabi, Libya. In: Neogene Paleontology and geology of Sahabi (Alan R. Liss ed.), 233-254.

Deraniyagala, P.E.P. (1951). A hornless rhinoceros from the Mio-Pliocene deposits of East Africa. Spolia Zeylanica, Colombo, 26: 133-135.

Gaudry, A. (1862-67). Animaux fossiles et géologie de l'Attique. F. Savy, Paris, 177-214.

Gentry, A.W. (1987). Rhinoceroses from the Miocene of Saudi Arabia. Bulletin of the British Museum of natural History (Geology), 41, 4: 409-432.

Geraads, D. (1986). Sur les relations phylétiques de Dicerorhinus primaevus Arambourg, 1959, rhinocéros du Vallésien d'Algérie. Comptes rendus hebdomadaires des séances de l'Académie des Sciences de Paris, II, 302 (13): 835-837.

Geraads, D. (1988). Révision des Rhinocerotinae (Mammalia) du Turolien de Pikermi. Comparaison avec les formes voisines. Annales de Paléontologie, Paris, 74, 1: 13-41.

Geraads, D. (1989). Vertébrés fossiles du Miocène supérieur du Djebel Krechem el Artsouma (Tunisie centrale). Comparaisons biostratigraphiques. Geobios, Lyon, 22 (6), 777-801.

Geraads, D. (2005). Pleistocene Rhinocerotidae (Mammalia) from Hadar and Dikika (Lower Awash, Ethiopia) and a revision of the origin of modern African rhinos. Journal of Vertebrate Paleontology, 25 (2): 451-461.

Geraads, D. \& Koufos, G. (1990). Upper Miocene Rhinocerotidae (Mammalia) from Pentalophos-1, Macedonia, Greece. Palaeontographica, Stuttgart, A, 210: 151-168.

Ginsburg, L. (1974). Les Rhinocérotidés du Miocène de Sansan (Gers). Comptes Rendus de l'Académie des Sciences de Paris, 278, D: 597-600.

Gloger, C.W.L. (1841). Gemeinnütziges Hand-und Hilfsbuch der Naturgeschichte. Aug. Schultz \& Comp., Breslau, 495 pp.

Gray, J. E. (1821). On the natural arrangement of vertebrose animals. London Medical Repository, 15 (1): 296-310.
Gray, J.E. (1868). Observations on the preserved specimens and skeletons of the Rhinocerotidae in the collection of the British Museum and Royal College of Surgeons, including description of three news species. Proceedings of the Zoological Society of London 1867: 1003-1032.

Groves, C.P. (1983). Phylogeny of the living species of Rhinoceros. Zeitschrift für zoologische Systematik und Evolutionsforschung, Hamburg, 21, 4: 293-313.

Guérin, C. (1966). Diceros douariensis nov. sp., un rhinocéros du Mio-Pliocène de Tunisie du Nord. Documents du Laboratoire de Géologie de la Faculté des Sciences de Lyon, 16: 1-50.

Guérin, C. (1976). Les restes de rhinocéros du gisement miocène de Béni-Mellal, Maroc. Géologie méditerranéenne, Marseille, 3 (2) :105-108.

Guérin, C. (1979). Chalicotheriidae et Rhinocerotidae (Mammalia, Perissodactyla) du Miocène au Pléistocène de la Rift Valley (Afrique orientale). Un exemple d'évolution: le squelette post-cranien des Diceros et Ceratotherium plio-pléistocènes. Bulletin de la Société géologique de France, 21 (3): 283-288.

Guérin, C. (1980a). A propos des rhinocéros (Mammalia, Perissodactyla) néogènes et quaternaires d'Afrique: essai de synthèse sur les espèces et sur les gisements. Proceedings 8th Panafrican Congress Prehistory and Quaternary studies, Nairobi 1977, p. 58-63.

Guérin, C. (1980b). Les rhinocéros (Mammalia, Perissodactyla) du Miocène terminal au Pléistocène supérieur en Europe occidentale. Comparaison avec les espèces actuelles. Documents des Laboratoires de Géologie de Lyon, 79: 1185 pp.

Guérin, C. (1985a). Les rhinocéros et les chalicothères (Mammalia, Perissodactyla) des gisements de la vallée de l'Omo en Ethiopie. (Collections françaises). In: Les faunes plio-pléistocènes de la basse vallée de l'Omo (Ethiopie) (Coppens, Y. \& Howell, F.C., eds.), t. 1, Cahiers de paléontologie - Travaux de paléontologie est-africaine, CNRS, Paris, 67-95.

Guérin, C. (1985b). Signification paléoécologique des Rhinocerotidae et Chalicotheriidae (Mammalia, Perissodactyla) des gisements à Hominidés du plio-pléistocène d'Afrique. Colloque internat. Fondation SingerPolignac, L'environnement des Hominidés au pliopléistocène, Paris, Juin 1981, Masson, Paris: 45-55.

Guérin, C. (1987). Fossil Rhinocerotidae (Mammalia, Perissodactyla) from Laetoli. In: Laetoli, a Pliocene site in Northern Tanzania (Leakey,M.D. \& Harris, J.M., eds.) Clarendon Press, Oxford: 320-348.

Guérin, C. (1989). La famille des Rhinocerotidae (Mammalia, Perissodactyla): systématique, histoire, évolution, paléoécologie. Cranium, Rotterdam, 6 (2): 3-14.

Guérin, C. (1994). Les Rhinocéros (Mammalia, Perissodactyla) du Néogène de l'Ouganda. In: Geology and Palaeobiology of the Albertine Rift Valley, UgandaZaire, (Senut, B. \& Pickford, M., eds.), vol. II: Palaeobiology, CIFEG édit., Orléans, occasional publication 1994/29: 263-279.

Guérin, C. (1999). Chapitre 5: l'évolution des faunes. In: Comment l'homme? A la découverte des premiers 
Hominidés d'Afrique de l'Est (Gallay, A., ed.). Errance, Paris, 143-198, 381-385.

Guérin, C. (2000). The Neogene Rhinoceroses of Namibia. Palaeontologia africana, 36: 119-138.

Guérin, C. (2003). Miocene Rhinocerotidae of the Orange River Valley, Namibia. In: Geology and palaeobiology of the Central and Southern Namib, vol. 2: Palaeontology of the Orange River Valley (Pickford, M. \& Senut, B., eds.), Geological Survey of Namibia, Windhoek, Memoir 19, 257-281.

Guérin, C. (2008). The Miocene Rhinocerotidae (Mammalia) of the Northern Sperrgebiet, Namibia. In: Geology and palaeobiology of the Northern Sperrgebiet, Namibia (Pickford, M. \& Senut, B., eds.). Memoir Geological Survey of Namibia, Windhoek, mem. 20, 331-341.

Guérin, C. \& Faure, M. (1983). Paléofaunes: Mammifères. In: Sahara ou Sahel? Quaternaire récent du bassin de Taoudenni (Mali) (Petit-Maire, N \& Riser, J., eds.), CNRS, Marseille, 239-260.

Guérin, C. \& Pickford, M. (2003). Ougandatherium napakense nov. gen. nov. sp., le plus ancien Rhinocerotidae Iranotheriinae d'Afrique. Annales de Paléontologie, Paris, 89, 1: 1 - 35.

Heissig, K. (1971). Brachypotherium aus dem Miozän von Südwestafrika. Mitteilungen der Bayerische Staatssammlung für Paläontologie und historische Geologie, München, 11: 125-128.

Heissig, K. (1975). Rhinocerotidae aus dem Jungtrertiär Anatoliens. Geologisches Jahrbuch, Hannover, B, 15: 145-151.

Heissig, K. (1982). Note on Sahabi Rhinocerotidae. Garyounis Scientific Bulletin, Benghazi, special issue, 4: 85.

Hooijer, D.A. (1946). Notes on some Pontian Mammals from Sicily, figured by Seguenza. Archives néerlandaises de Zoologie, 7: 301-333.

Hooijer, D.A. (1958). Fossil Rhinoceroses from the Limeworks Cave, Makapansgat. Palaeontologia Africana, 6: 1- 13.

Hooijer, D.A. (1963). Miocene Mammalia of Congo (with a chapter by A. Gautier and J. Lepersonne). Annales du Musée Royal de l'Afrique centrale, Tervueren, Sci. Géol. 46: 1-77.

Hooijer, D.A. (1966). Miocene rhinoceroses of East Africa. Bulletin of the British Museum (Natural History), London, 13 (2): 119-190.

Hooijer, D.A. (1968 a). A note on the mandible of Aceratherium acutirostratum (Deraniyagala) from Moruaret Hill, Turkana District, Kenya. Zoologische Mededelingen, Leiden, 42 (21): 231-235.

Hooijer, D.A. (1968 b). A rhinoceros from the late Miocene of Fort Ternan, Kenya. Zoologische Mededelingen, Leiden, 43 (6): 77-92.

Hooijer, D.A. (1971). A new rhinoceroses from the late Miocene of Loperot, Turkana District, Kenya. Bulletin of the Museum of Comparative Zoology, Cambridge (Mass.), 142 (3): 339-392.

Hooijer, D.A. (1973). Additional Miocene to Pleistocene rhinoceroses of Africa. Zoologische Mededelingen, Leiden, 46 (11): 149-178.

Hooijer, D.A. (1978). 19: Rhinocerotidae. In: Evolution of African Mammals (Maglio, V.J. \& H.B.S. Cooke, H.B.S., eds.), Harvard University Press, Cambridge (Mass.), 371-378.

Hooijer, D.A. \& Singer, R. (1960). Fossil rhinoceroses from Hopefield, South Africa. Zoologische Mededelingen, 37 (8): 113-128.

Kaup, J.J. (1832). Description d'ossements fossiles de Mammifères inconnus jusqu'à-présent, qui se trouvent au Muséum grand-ducal de Darmstadt. J.G. Heyer, Darsmstadt, 49-61.

Likius, A. (2002). Les grands ongulés du Mio-Pliocène $d u$ Tchad (Rhinocerotidae, Giraffidae, Camelidae): systématique, implications paléobiogéographiques et paléoenvironnementales. Thèse Université de Poitiers, $188 \mathrm{pp}$.

Nakaya, H.; Pickford, M.; Yasui, K. \& Nakano, Y. (1999, daté de 1987). Additional large mammalian fauna from the Namurungule Formation, Samburu Hills, Northern Kenya. African Study Monograph,s Suppl. Issue. 5: 79-130.

Pickford, M.; Senut, B. \& Hadoto, D. (1993). Geology and Palaeobiology of the Albertine Rift Valley, Uganda-Zaire, vol. I: Geology, CIFEG édit, Orléans, occasional publication 1993/24, 190 pp.

Pickford, M. \& Senut, B. (2001). The geological and faunal context of Late Miocene hominid remains from Lukeino, Kenya. Comptes rendus de l'Académie des Sciences de Paris, Sciences de la Terre et des planètes, 332: $145-152$.

Roger, O., 1904: Wirbelthierreste aus dem Obermiocän der bayerisch-schwabischen Hochebene. Bericht des Naturwissenschaftlichen Vereins für Schwaben und Neuburg, 36: 1-22.

Senut, B.; Pickford, M.; Gommery, D.; Mein, P.; Cheboi, K. \& Coppens, Y. (2001). First hominid from the Miocene (Lukeino Formation, Kenya). Comptes rendus de l'Académie des Sciences de Paris, Sciences de la Terre et des planètes, 332: 137-144.

Thenius, E. (1955). Zur Kenntniss der unterpliozänen Diceros-Arten (Mammalia, Rhinocerotidae). Annalen des Naturhistorischen Museums Wien: 60: 202-209.

Thenius, E. (1956). Über das Vorkommen von Diceros pachygnathus im Pannon (Unter-Pliozän) des Wiener Beckens. Neues Jahrbuch für Geologie und Paläontolologie, Monatshefte, 1: 35-39.

Recibido el 25 de marzo de 2011 Aceptado el 9 de septiembre de 2011 